\title{
Torgau 1519 \\ Der bayerische Adlige Hans Herzheimer beschreibt die kursächsische Residenz
}

\author{
von \\ ENNO BÜNZ
}

Torgau beeindruckt als Stadt der Renaissance durch die in hervorragender Geschlossenheit erhaltene Bausubstanz seine Besucher immer wieder. ${ }^{1}$ Als ein Zentrum der Reformation ist die kursächsische Residenzstadt nicht nur den Kennern der Landesgeschichte ein Begriff, sondern weit über Sachsen hinaus bekannt. ${ }^{2}$ Lucas Cranach d. Ä. vermittelt zudem durch eine Reihe von Jagdbildern eine recht anschauliche Vorstellung des Aussehens der Stadt vor fast 500 Jahren. $^{3}$ In Torgau wird deshalb wie in keiner anderen sächsischen Stadt die Welt des frühen 16. Jahrhunderts lebendig, aus der sich die Reformation entwickelt hat.

Die Straßenzüge und Wohnhäuser, die Kirchen und Schlösser helfen, sich die Welt um 1500 besser vorzustellen, aber sie bleiben doch letztlich stumm, wenn wir keine zeitgenössischen Quellen heranziehen können, die näher an das Leben

1 Grundlegend zum Baubestand Peter Findeisen/Heinrich Magirius (Bearb.), Denkmale der Stadt Torgau (Die Denkmale im Bezirk Leipzig), Leipzig 1976. Ergänzend die Beiträge in: Das kurfürstliche Kanzleihaus zu Torgau. Erkenntnisse zur Bau- und Nutzungsgeschichte (Schriften des Torgauer Geschichtsvereins 2), Torgau 2001; und in: Tilmann von Stockhausen (Hg.), Torgau - Stadt der Renaissance, Dresden 2003; Jürgen Herzog, Torgau - Eine Renaissancestadt stellt sich vor, in: Lutherstadt Wittenberg, Torgau und der Hausbau im 16. Jahrhundert (Jahrbuch für Hausforschung 62), Marburg 2015, S. 191-205.

2 Eine umfassende Stadtgeschichte fehlt. Knappe Abrisse bieten Martin Granzin/ Berent Schwineköper, Torgau, in: Berent Schwineköper (Hg.), Provinz SachsenAnhalt (Handbuch der historischen Stätten Deutschlands 11), Stuttgart 21987, S. 467470; und Reinhardt Butz, Torgau, in: Werner Paravicini (Hg.), Höfe und Residenzen im spätmittelalterlichen Reich. Ein dynastisch-topographisches Handbuch, Teilband 2: Residenzen (Residenzenforschung 15.I/2), Ostfildern 2003, S. 582-584, wo die wichtigste ältere Literatur genannt wird. Siehe auch die Beiträge in: MARTIN BRECHT/HANSJOCHen Hancke (Hg.), Torgau. Stadt der Reformation. Luthers Torgauer Kirchweihe 1544. 450-Jahr-Feier der Einweihung der Schlosskirche auf Schloss Hartenfels, Torgau 1996; und in: Glaube und Macht. Sachsen im Europa der Reformationszeit. 2. Sächsische Landesausstellung Torgau, Schloss Hartenfels 2004, Katalog, hrsg. von Harald Marx/ Eckhard Kluth, Aufsätze, hrsg. von Harald Marx/Cecilie Hollberg, Dresden 2004; Jürgen Herzog, Beiträge zur Residenz- und Reformationsgeschichte der Stadt Torgau (Schriften des Torgauer Geschichtsvereins 8), Beucha 2015.

3 Hansjochen Hancke, Das Torgauer Stadtbild und die Ansichten von Lucas Cranach, in: Stockhausen, Torgau (wie Anm. 1), S. 48-57. Vgl. auch Ders., Torgau - Stadtbild im 16. Jahrhundert, in: Brecht/Hancke, Torgau (wie Anm. 2), S. 51-59. 
heranführen. Aus Fernsehdokumentationen zur jüngsten Geschichte ist es uns selbstverständlich geworden, dass zu allen erdenklichen Themen Zeitzeugen berichten, und manchmal gewinnt man schon den Eindruck, dass es gar keine anderen Wege in die Geschichte mehr gibt. ${ }^{4}$

Für die älteren Epochen besteht zwar nicht mehr die Möglichkeit, Zeitzeugen sprechen zu lassen, aber es gibt doch manche Selbstzeugnisse beziehungsweise Ego-Dokumente, die von hoher Authentizität sind. Zu ihnen gehören auch Reiseberichte und -tagebücher, wie sie uns seit dem späten Mittelalter in wachsender Zahl überliefert sind. Durchmustert man die einige hundert Berichte, die von europäischen Reisenden der Zeit bislang bekannt geworden sind, zeigt sich allerdings der etwas ernüchternde Befund, dass die allermeisten Aufzeichnungen anlässlich von Pilgerreisen nach Jerusalem und Santiago de Compostela angefertigt wurden. Über andere Reisen wurde hingegen nur selten berichtet. ${ }^{5}$

Vor diesem Hintergrund ist der Reisebericht, der hier ausgewertet werden soll, ein ganz besonderer Glücksfall. Torgau hat als ein bedeutender Residenzort der Kurfürsten von Sachsen gewiss viele Reisende angezogen, ${ }^{6}$ aber sie haben darüber keine Aufzeichnungen hinterlassen. Eine Ausnahme stellt der bayerische Adlige Hans III. Herzheimer (1464-1532) dar, der 1518/19 eine mehrmonatige Reise durch Sachsen unternommen und darüber ein Tagebuch geführt hat.7 Ein noch größerer Glücksfall ist es wohl, dass dieser tagebuchartige Bericht auch noch erhalten geblieben ist. Die einzige Handschrift des Herzheimer-Berichts befindet sich in der Bibliothek des MAK - Österreichisches Museum für angewandte Kunst / Gegenwartskunst in Wien, wohin sie 1930 durch Versteigerung der

4 Dazu auch mit kritischen Beiträgen Martin SAbrow/Norbert Frei (Hg.), Die Geburt des Zeitzeugen nach 1945 (Geschichte der Gegenwart 4 = Beiträge zur Geschichte des 20. Jahrhunderts 14), Göttingen 2012.

5 Einen guten Überblick der bislang gedruckten Reiseberichte bietet WernER PARAVICINI (Hg.), Europäische Reiseberichte des späten Mittelalters. Eine analytische Bibliographie, Teil 1: Deutsche Reiseberichte, bearb. von Christian Halm (Kieler Werkstücke D/5), Frankfurt a. M. u. a. ${ }^{2} 2000$, Teil 2: Französische Reiseberichte, bearb. von Jörg Wettlaufer in Zusammenarbeit mit Jacques Paviot (Kieler Werkstücke D/12), Frankfurt a. M. u. a. 1999, Teil 3: Niederländische Reiseberichte, nach Vorarbeiten von Detlev Kraack bearb. von Jan Hirschbiegel (Kieler Werkstücke D/14), Frankfurt a. M. u. a. 2000; siehe dazu EnNo Bünz, Reiseberichte - Reisegruppen - Reisewege. Bemerkungen zur neuen analytischen Bibliographie „Europäische Reiseberichte des späten Mittelalters“, in: Würzburger Diözesangeschichtsblätter 65 (2003), S. 353-361; WinfrIED MüLleR, Sachsen und der mitteldeutsche Raum in Reiseberichten der Frühen Neuzeit. Bibliografie und Ortserschließung, in: Neues Archiv für sächsische Geschichte 83 (2012), S. 35-92.

6 Zur Residenzfunktion zuletzt Jürgen Herzog, Fürstlicher Hof und Stadt Torgau während der Regierungszeit Friedrichs des Weisen, in: Armin Kohnle/Uwe Schirmer (Hg.), Kurfürst Friedrich der Weise von Sachsen. Politik, Kultur und Reformation (Quellen und Forschungen zur sächsischen Geschichte 40), Leipzig/Stuttgart 2015, S. 270-328; wieder abgedruckt in: Ders., Beiträge zur Residenz- und Reformationsgeschichte (wie Anm. 2), S. 9-72; Ders., Die Stadt Torgau am Vorabend zur bevorzugten kurfürstlichen Residenz (1531), in: Sächsische Heimatblätter 55 (2009), S. 336-354.

7 Siehe unten Anm. 13. 
Sammlung des österreichischen Bankiers Albert Figdor (1843-1927) 8 in Wien gelangt ist. Es ist das Verdienst des Grazer Kirchenhistorikers Karl Amon, die Handschrift vor etlichen Jahrzehnten der Vergessenheit entrissen und in der Bibliothek des MAK wiederaufgefunden zu haben. ${ }^{9}$ Amon hat nur den Bericht über Wittenberg 1980 in Auszügen publiziert beziehungsweise paraphrasiert und kommentiert. ${ }^{10}$ Aufgrund seiner Mitteilungen konnte die Leipziger Kirchenhistorikerin Ingetraut Ludolphy in ihrer Biografie Kurfürst Friedrichs des Weisen einige Auszüge aus dem Reisetagebuch Herzheimers zitieren. ${ }^{11}$ Der Kunsthistoriker Stefan Hoppe publizierte vor zehn Jahren Herzheimers Beschreibung der Residenz Lochau (heute Annaburg). ${ }^{12}$ Seit einigen Jahren bin ich nun mit der Edition des Reiseberichts beschäftigt. In diesen Zusammenhang gehört die vorliegende Untersuchung. ${ }^{13}$ Die kommentierte Edition wird 2017 als Buch erscheinen. ${ }^{14}$

Hans Herzheimer stammt aus einer niederadligen Familie, die im Herzogtum Niederbayern ansässig war. Ihr Stammsitz Heretsham liegt bei Trostberg an der Alz im Landkreis Traunstein (heute Oberbayern). Die Burg ist abgegangen.

8 Gustav Otruba, Albert Figdor, in: Neue Deutsche Biographie 5 (1961), S. 143 f., Onlinefassung: https://www.deutsche-biographie.de. Vor Figdors Tod hatte die Handschrift noch Jakob Weichselgartner benutzen können, siehe Jаков WeichSELGARTNER, Salmanskirchen. Geschichtlicher Überblick, in: Der Inn-Isengau 5 (1927), S. 10-15, 3648, 86-97, hier S. 45, Anm. 2 mit Hinweis auf das „Reisetagebuch“.

9 Museum für angewandte Kunst Wien (im Folgenden: MAK Wien), Inv. B.I. 21.517, Standort S20; zur Handschrift Karl Amon, Hans Herzheimer in Wittenberg 1518/19, in: Remigius Bäumer (Hg.), Reformatio Ecclesiae. Beiträge zu kirchlichen Reformbemühungen von der Alten Kirche bis zur Neuzeit. Festgabe für Erwin Iserloh, Paderborn u. a. 1980, S. 301-319, hier S. 302 f.

10 Verbesserter und kommentierter Abdruck nun von EnNo BüNZ, Wittenberg 1519: Was ein Reisender von der Stadt wahrgenommen hat, und was nicht. Mit einer Teiledition der Aufzeichnungen Hans Herzheimers, in: Heiner Lück u. a. (Hg.), Das ernestinische Wittenberg: Stadt und Bewohner (Wittenberg-Forschungen 2.1-2), Petersberg 2013, Textband S. 9-24, Bildband S. 15-18.

11 Ingetraut Ludolphy, Friedrich der Weise. Kurfürst von Sachsen 1463-1525, Göttingen 1984, S. 529 (Literaturverzeichnis); die einschlägigen Zitate lassen sich über das Register S. 584 s. v. Hans Herzheimer ermitteln. Neben Ludolphy ist stets die quellenfundierte Dissertation von BERnd STEPHAN, „Ein itzlichs Werck lobt seinen Meister“. Friedrich der Weise, Bildung und Künste (Leucorea-Studien zur Geschichte der Reformation und der Lutherischen Orthodoxie 24), Leipzig 2014, zu benutzen.

12 Stephan Hoppe, Anatomy of an Early „Villa“ in Central Europe. The Schloss and Garden of the Saxon Elector Frederick the Wise in Lochau (Annaburg) according to the 1519 Report of Hans Herzheimer, in: Monique Chatenet (Hg.), Maisons des champs dans l'Europe de la Renaissance. Actes des premières Rencontres d'architecture européenne, Château de Maisons, 10-13 juin 2003, Paris 2006, S. 159-170.

13 Zum Herzheimer-Bericht siehe bisher Bünz, Wittenberg 1519 (wie Anm. 10); Ders., Die Wettiner auf den Reichstagen: Kurfürst Friedrich der Weise auf dem Wahltag 1519 in Frankfurt, gesehen mit den Augen eines Zeitzeugen, in: Oliver Auge (Hg.), König, Reich und Fürsten im Mittelalter (Beiträge zur Geschichte der Universität Greifswald 12), Stuttgart 2017, S. 437-456 (im Druck).

14 Der Leiterin von Bibliothek und Kunstblättersammlung/Archiv des MAK Wien, Frau Mag. Kathrin Pokorny-Nagel, habe ich schon jetzt sehr herzlich für die vertrauensvolle Zusammenarbeit und die förderliche, kontinuierliche Unterstützung zu danken. 
Hans III. Herzheimer dürfte die wohl bedeutendste und in jedem Fall am besten dokumentierte Gestalt sein, die aus dieser Niederadelsfamilie hervorgegangen ist. ${ }^{15}$ Schon dank seiner eigenen Aufzeichnungen, aber auch aufgrund der vielfältigen Spuren, die Hans Herzheimer auf seinem Lebensweg auch in Gestalt von Grab- und Gedenksteinen hinterlassen hat, eröffnen sich ungewöhnlich anschauliche und dichte Einblicke in Denkart und Existenz eines bayerischen Niederadligen an der Wende vom Mittelalter zur Neuzeit. Geboren wurde er am 27. Dezember 1464 als Sohn des Georg Herzheimer und dessen Gemahlin Veronika, einer geborenen Pfeffinger. Veronika wiederum war eine Schwester des Degenhart Pfeffinger (1471-1524), dessen Familie das Erbmarschallamt des Herzogtums Niederbayern innehatte. Pfeffinger stieg zunächst im Dienste der niederbayerischen Herzöge von Bayern-Landshut auf, nahm 1493 mit anderen bayerischen Adligen an der Pilgerfahrt Kurfürst Friedrichs des Weisen von Sachsen in das Heilige Land teil und wechselte danach in dessen Dienste. ${ }^{16}$ Zunächst als „Türknecht“, womit eine Verwaltungsfunktion gemeint ist, dann als Landrentmeister und als ernestinischer Rat gehörte Pfeffinger zu den engsten Vertrauten des sächsischen Kurfürsten. Wir werden ihm auch in Torgau noch wiederbegegnen. Zweifellos war es Degenhard Pfeffinger, der seinem Neffen Hans III. Herzheimer auf dessen Reise durch Sachsen viele Türen am kurfürstlichen Hof öffnete. Andreas Meinhardi rühmt in seiner 1508 gedruckten Beschreibung der Stadt Wittenberg Degenhard Pfeffinger als treuen und in jeder Hinsicht umsichtigen Diener Friedrichs des Weisen und betont: „Wem er den Zutritt zum Hof des erlauchten Fürsten

15 Von der älteren Literatur sei hier nur verwiesen auf ERnEsT Geiss, Geschichte des Schlosses Herzheim und seiner Bewohner, in: Oberbayerisches Archiv für vaterländische Geschichte 7 (1846), S. 186-237, hier S. 202-221 über Hans III. Herzheimer und seine Kinder. Grundlegend, aber leider ungedruckt sind die Werke von Јаков WeіснSelgartner, Die Herzheimer von Herzheim, 1962; und Ders., Hans III. von Hertzheim, o. J. Beide Typoskripte befinden sich im Nachlass des Geistlichen, der in der Bibliothek des Metropolitankapitels München verwahrt wird. Von neueren Arbeiten ist vor allem zu erwähnen Ferdinand Tremel, Hans Herzheimer, Aussee, und die Ausseer Hallamtsordnung vom Jahre 1513, in: Alexander Novotny/Othmar Pickl (Hg.), Festschrift Hermann Wiesflecker zum sechzigsten Geburtstag, Graz 1973, S. 8197. Siehe künftig auch EnNo Bünz, „ich Hans Herzheimer“. Ein Adliger der Maximilianszeit in Bad Aussee, Bayern und Europa (1464-1532), in: Zeitschrift für bayerische Landesgeschichte (in Vorbereitung).

$16 \mathrm{Zu}$ seiner Biografie ENNO BüNZ, Die Heiltumssammlung des Degenhard Pfeffinger, in: Andreas Tacke (Hg.), „Ich armer sundiger mensch“. Heiligen- und Reliquienkult am Übergang zum konfessionellen Zeitalter (Schriftenreihe der Stiftung Moritzburg, Kunstmuseum des Landes Sachsen-Anhalt 2), Göttingen 2006, S. 125-169, hier S. 132138 und S. 162-167, wo die ältere Literatur genannt wird. Zu einer Porträtmedaille Degenhard Pfeffingers, die zwischen 1505 und 1511 wohl am kursächsischen Hof entstanden ist, siehe jüngst Walter Cupperi/Martin Hirsch/Annette Kranz/Ulrich Pfisterer (Hg.), Wettstreit in Erz. Porträtmedaillen der deutschen Renaissance, Berlin/ München 2013, S. 148 (Katalogartikel von Charles Davis) mit Abb., doch leider ohne Kenntnis des wichtigen Aufsatzes von Karl Koetschau, Die Medaille auf Degenhard Pfeffinger, in: Zeitschrift für Numismatik 20 (1897), S. 310-324. 
gestattet, dem stehen Tore und Türen ohne Zweifel weit offen “.17 Da die Pfeffinger mit Degenhard 1519 ausstarben, gelangte das Erbe über Degenhards Schwester Veronika, die mit Georg Herzheimer verheiratet war, an ihren Sohn Hans III. Herzheimer. ${ }^{18}$

Hans III. Herzheimer war seit 1494 zweiter, seit 1499 erster Verweser des Hallamtes im Markt Aussee in der Steiermark. ${ }^{19}$ Aus seiner Ehe mit Ehrentrudis, die aus einer Salzburger Bürgerfamilie stammte, gingen drei Söhne hervor, von denen Georg seit 1514 in Ingolstadt studierte, während die beiden älteren Söhne Johannes Evangelist und Johannes Jordan Baptist bereits 1512 die Universität Wittenberg bezogen hatten. ${ }^{20}$ Dabei ist anzunehmen, dass Degenhard Pfeffinger Herzheimer angeregt hatte, seine Söhne nach Wittenberg zu schicken. Die studierenden Söhne nach jahrelanger Trennung wiederzusehen, war der eigentliche Anlass der Reise Hans Herzheimers nach Kursachsen.

Am 14. Dezember 1518 brach Hans Herzheimer gemeinsam mit seinem Vetter Degenhard Pfeffinger zu Pferd von Salmanskirchen nach Sachsen auf. Sie hatten noch das Eintreffen des päpstlichen Legaten Karl von Miltitz (1490-1529) abgewartet, welcher wegen der Luther-Sache zum sächsischen Kurfürsten unterwegs war. ${ }^{21}$ Im Reisegepäck führte er u. a. Briefe des päpstlichen Vizekanzlers Kardinal

17 Andreas Meinhardi, Dialogus illustrate ac Augustissime vrbis Albiorene vulgo Vittenberg dicte Situm Amenitatem ac Illustrationem docens [...], Leipzig 1508 (VD 16, M 2251), ediert von Edgar C. Reinke, The Dialogus of Andreas Meinhardi. A Utopian Description of Wittenberg and Its University, Ann Arbor 1976, mit englischer Übersetzung; auf dieser Grundlage die deutschsprachige Ausgabe Andreas MeINHARDI, Über die Lage, die Schönheit und den Ruhm der hochberühmten herrlichen Stadt Albioris, gemeinhin Wittenberg genannt. Ein Dialog, herausgegeben für diejenigen, die ihre Lehrzeit in den edlen Wissenschaften beginnen. Aus dem Lateinischen. Ubersetzung, Einleitung und Anmerkungen von Martin Treu (Reclams Universal-Bibliothek 1145), Leipzig 1986, hier S. 89. Zu Verfasser und Werk nun Klaus J. Kipf, Meinhardi, Andreas, in: Franz Josef Worstbrock (Hg.), Deutscher Humanismus 1480-1520. Verfasserlexikon, Band 2, Berlin u. a. 2013, Sp. 209-213.

18 Am Stammsitz Degenhard Pfeffingers in Salmanskirchen bei Mühldorf am Inn (Oberbayern) ließ Hans III. von Herzheim einen prächtigen Gedenkstein aus Rotmarmor anbringen, der sich heute im Bayerischen Nationalmuseum in München befindet und in einer ausführlichen deutschen Inschrift von 1520 an den früheren Besitzer des Schlosses, Degenhard Pfeffinger, und an dessen Erben Hans Herzheimer erinnert. Die Inschrift umrahmt drei durch Rundbogen unterteilte Bildfelder: in der Mitte das Stammwappen der Herzheimer, vom Betrachter links im Profil ein Mann mit pelzverbrämter Schaube, mit Haube und Schulterkette, gewiss Degenhard Pfeffinger darstellend, rechts ein Ritter in Plattenharnisch mit Federhut, bei dem es sich um Hans III. Herzheimer handeln dürfte. Siehe die Beschreibung mit Abbildung und Transkription der Inschrift in: Theodor Müller (Bearb.), Die Bildwerke in Holz, Ton und Stein von der Mitte des XV. bis gegen Mitte des XVI. Jahrhunderts (Kataloge des Bayerischen Nationalmuseums München XIII.2), München 1959, S. 227 f., Nr. 232.

Tremel, Hans Herzheimer (wie Anm. 15), S. 84 f.

20 Die Lebensdaten nach Weichselgartner, Salmanskirchen (wie Anm. 8), S. 46 f.

21 Siehe die Hinweise bei Amon, Hans Herzheimer (wie Anm. 9), S. 305; Martin BRECHT, Martin Luther, Band 1: Sein Weg zur Reformation 1483-1521, Stuttgart ${ }^{3} 1990$, S. 255-263; Ludolphy, Friedrich der Weise (wie Anm. 11), S. 411-414. Die positive 
Julius Medici und ein Breve Papst Leos X. vom 24. Oktober 1518 mit sich, in denen Degenhard Pfeffinger aufgefordert wurde, gegen die lutherische Ketzerei vorzugehen. ${ }^{22}$ Die Reiseaufzeichnungen Hans Herzheimers gehen aber auf den von Luther initiierten Ablassstreit und die diesbezüglichen Interessenlagen von Kurie und Landesherrn nicht weiter ein. ${ }^{23}$ Für die Gesamtperspektive des Reisetagebuchs ist das bezeichnend. Das große Zeitgeschehen - sieht man einmal von den Aufzeichnungen über den Tod Kaiser Maximilians I. am 12. Januar 1519 in Wels ${ }^{24}$ und die Wahl Karls V. am 28. Juni 1519 in Frankfurt am Main ${ }^{25} \mathrm{ab}$ - wird auch sonst kaum wahrgenommen. Wie Forschungen über Jerusalempilgerberichte des späten Mittelalters zeigen, sind die Wahrnehmungen von Reisenden zumeist stereotyp und selektiv. ${ }^{26}$ Dies gilt auch für Stadtbeschreibungen dieser Zeit, die auf der einen Seite traditionellen Darstellungsmustern verpflichtet sind, auf der anderen Seite aber auch den intellektuell und sozial bestimmten Wahrnehmungshorizont der Reisenden sowie individuell bestimmte Sichtweisen und Interessen der Reisenden widerspiegeln. ${ }^{27}$ Ein adliger Reisender war naheliegender Weise auf den Hof und die Residenz fixiert. Dies wird in den Aufzeichnungen Hans Herzheimers recht deutlich, zumal er am kursächsischen Hof besondere Zugangsmöglichkeiten hatte.

Deshalb nimmt es nicht wunder, dass Hans Herzheimer in seinem Reisebericht ausführlich auf die drei wichtigsten Residenzorte Kurfürst Friedrichs des Weisen

Neubewertung seiner Rolle angedeutet bei Heribert Smolinsky, Karl von Miltitz, in: Neue Deutsche Biographie 17 (1994), S. 533 f.

22 Bünz, Heiltumssammlung (wie Anm. 16), S. 137 f. mit Anm. 58, wo sich weiterführende Hinweise finden.

23 Auch Martin Luther selbst wird übrigens nur einmal genannt, als Herzheimer während seines Aufenthaltes in Wittenberg das dortige Augustinerkloster erwähnt. Siehe BüNZ, Wittenberg 1519 (wie Anm. 10), S. 22 f.

24 Dieser Abschnitt ediert von Hanna Dornik-Eger, Albrecht Dürer und die Druckgraphik für Kaiser Maximilian I., Wien 1971; Sie hat S. 24-31 „Hans Herzheimers ,Neue Zeitung' zum Tode Kaiser Maximilians I.“ kommentiert und S. 33-38 ediert. Siehe auch unten im Editionsanhang Abschnitt Nr. 118.

25 Dazu nun BüNZ, Wettiner auf den Reichstagen (wie Anm. 13), passim.

26 Ursula Ganz-Blättler, Andacht und Abenteuer. Berichte europäischer Jerusalemund Santiago-Pilger (1320-1520) (Jakobus-Studien 4), Tübingen 32000; FolKer Reichert, Asien und Europa im Mittelalter: Studien zur Geschichte des Reisens, Göttingen 2014; siehe auch die Arbeiten von Arnold Esch, unten Anm. 103.

27 Vgl. Gerhard Fouquet, Mit dem Blick des Fremden. Stadt und Urbanität in der Wahrnehmung spätmittelalterlicher Reise- und Stadtbeschreibungen, in: Ferdinand Opll (Hg.), Bild und Wahrnehmung der Stadt (Beiträge zur Geschichte der Städte Mitteleuropas 19), Linz 2004, S. 45-65; Klaus Arnold, Städtelob und Stadtbeschreibung im späten Mittelalter und in der frühen Neuzeit, in: Peter Johanek (Hg.), Städtische Geschichtsschreibung im Spätmittelalter und in der frühen Neuzeit (Städteforschung A/47), Köln u. a. 2000, S. 247-268; Eberhard Isenmann, Die deutsche Stadt im Mittelalter 1150-1550. Stadtgestalt, Recht, Verfassung, Stadtregiment, Kirche, Gesellschaft, Wirtschaft, Köln/Weimar/Wien 2012, geht nur S. 26 f. und S. 35 f. auf die Wahrnehmung der spätmittelalterlichen Stadt in Reiseberichten ein. Siehe auch dort die Literaturangaben S. 1008-1011. 
von Sachsen eingeht: Wittenberg, Lochau (das heutige Annaburg) und Torgau. Sachsen war bekanntlich seit der Leipziger Teilung 1485 in das ernestinische Kurfürstentum und das albertinische Herzogtum Sachsen geteilt. Hans Herzheimer hat sich während seiner Rundreise 1518/19 fast nur im Kurfürstentum Sachsen aufgehalten. $\mathrm{Zu}$ den wenigen Abstechern ins albertinische Herzogtum gehören Annaberg, Freiberg und Wolkenstein. In Dresden und Meißen ist Hans Herzheimer hingegen nie gewesen. Die vorliegende Untersuchung soll nicht nur auf den historisch-landeskundlichen Gehalt der Schilderungen Hans Herzheimers aufmerksam machen, sondern auch seine besondere Wahrnehmung herausarbeiten: Was hat er in den kursächsischen Residenzstätten gesehen und was nicht? Was hat ihn besonders interessiert, und was vielleicht auch gar nicht?

Folgen wir nun unserem Berichterstatter Hans Herzheimer sowie seinen Reisebegleitern, dem kursächsischen Kämmerer Degenhard Pfeffinger und dem päpstlichen Nuntius Karl von Miltitz, auf ihrem Weg nach Sachsen. Sie hatten sich, wie schon erwähnt wurde, am Stammsitz Pfeffingers in Salmanskirchen getroffen, und dort schloss sich ihnen neben dem päpstlichen Gesandten auch noch Herzheimers Vetter Sebastian an, der mit nach Sachsen reiten wollte, um in Dresden seinen Dienst am Hof Herzog Georgs anzutreten. Auch dieses Detail zeigt, dass um 1500 die Beziehungen zwischen Sachsen und Bayern eng waren.

Am 11. Dezember 1518 überschreibt Hans Herzheimer seinen Tagesbericht mit den Worten: „Ausgezogen nach Sachsen“.28 Von Kloster Frauenchiemsee ritt Herzheimer zunächst noch einmal zu seinem Familiensitz Heretsham, dann nach Salmanskirchen, von wo die Reisegruppe am 14. Dezember 1518 nach Sachsen aufbrach. Über Landshut, Neustadt an der Donau, Berching, Nürnberg, Bamberg und Coburg ging es zunächst nach Gräfenthal und Neustadt an der Orla, wo man den Heiligen Abend verbrachte, dann weiter nach Gera und Altenburg, das am 26. Dezember erreicht wurde. Dort blieb der päpstliche Gesandte Karl von Miltitz zurück, um im Haus des kurfürstlichen Sekretärs Georg Spalatin mit Martin Luther zu verhandeln. ${ }^{29}$ Hans Herzheimer und Degenhard Pfeffinger ritten am 6. Januar 1519 über Borna, Leipzig und Eilenburg weiter nach Torgau, wo sie am 9. Januar eintrafen. Es ist nach Altenburg die erste Reisestation, die Herzheimer ausführlicher beschreibt (Editionsanhang Nr. 43-53). Allerdings ist er zunächst nur kurz dort geblieben, denn bereits am nächsten Tag ritt Herzheimer nach Lochau weiter. ${ }^{30}$ Erst gegen Ende seiner Reise durch Sachsen, am 27. April, kam Hans Herzheimer nochmals nach Torgau, wo er diesmal gut drei Wochen zubrachte, weil er wegen seinem pösen bayn ruhebedürftig war (Editionsanhang Nr. 193-194). Erst am 23. Mai konnte Hans Herzheimer von Torgau nach Altenburg aufbrechen (Editionsanhang Nr. 196), um von dort im Gefolge des Kurfürs-

28 MAK Wien (wie Anm. 9), fol. 254v.

29 Paul Kalkoff, Die Miltitziade. Eine kritische Nachlese zur Geschichte des Ablaßstreites, Leipzig 1911; Irmgard Höss, Georg Spalatin 1484-1545. Ein Leben in der Zeit des Humanismus und der Reformation, Weimar 21989, S. 144 f. 
ten von Sachsen nach Frankfurt am Main zu reiten, wo im Juni 1519 die Wahl Kaiser Karls V. stattfinden sollte.

Am 9. Januar hat sich Hans Herzheimer Torgau von Eilenburg her genähert und dabei, wie er schreibt (Editionsanhang Nr. 43), einen „schönen Kiefern- und Eichenwald" durchritten, die Dübener Heide, und näherte sich von Südwesten her der Stadt. So sei er in ain vesste statt mit schudt ond polwerck ond wasser graben pevesstent khomen, genandt Torchaw. ${ }^{31}$ Neben einem Wassergraben war die Stadt demnach von Erdaufschüttungen und Bollwerken umgeben, doch bleibt unklar, $\mathrm{ob}$ es sich um einfache Erdaufschüttungen für Artillerie handelt, oder um vorgeschobene Erdrondelle mit flankierender Schusswirkung. Beides kommt bei Stadtbefestigungen des frühen 16. Jahrhunderts schon vor. ${ }^{32}$ Die Torgauer Stadtansichten des 16. und 17. Jahrhunderts lassen nicht erkennen, wie diese Befestigung angelegt war. Vermutlich hat Hans Herzheimer 1519 das Leipziger Tor passiert. ${ }^{33}$ Von den mächtigen Befestigungen zeigt sich Herzheimer auch bei anderen sächsischen Städten beeindruckt. Weiter hebt Herzheimer hervor, dass Torgau kurfürstliche Residenz sei: allda ir churf(urstlich) g(naden) auch sein hofflager bellt. „Hoflager“ ist der von Herzheimer durchgängig verwandte Begriff für „Residenz“. ${ }^{34}$

Die Beschreibung des Schlosses und der dazu gehörigen Bauten und Anlagen nimmt deshalb im Reisebericht Herzheimers auch den größten Raum ein (Editionsanhang Nr. 44-51). Das sloss in Torgaw schon erpauen ist der erste von fünf Abschnitten über die Residenz überschrieben. Der Begriff sloss kann in dieser Zeit den befestigten Wehrbau (Burg) ebenso wie den unbefestigten Repräsentationsbau (Schloss) bezeichnen. ${ }^{35}$ Es sei so wunder schon erpawen mit frembden ond auf ain new artt, der gleichen ich vor nye auf dise artt gesehen hab, doch räumt er kleinmütig ein, diss sloss gepewe khan ich nit aigentlich beschreiben. Konkret schildert er nur den bereits erwähnten Gang, der vom Schloss zur Pfarrkirche führte, und verweist auf die besondere Dachverzierung, nämlich etliche bochen knopfen, die vber alle dächer auf sindt gepawen (Editionsanhang Nr. 44). Hierbei mag es sich um Turmknöpfe oder -kugeln gehandelt haben. $\mathrm{Zu}$ denken ist aber auch an Dekormotive, die auf den Dächern der Zwerchhäuser bzw. Lukarnen angebracht waren. Als goldene Kugeln sind solche Verzierungen der Zwerchgiebel z. B. auf

31 Zur Stadtbefestigung Findeisen/Magirius, Denkmale der Stadt Torgau (wie Anm. 1), S. $723 \mathrm{f}$.

32 Thomas Biller, Die mittelalterliche Stadtbefestigung im deutschsprachigen Raum. Ein Handbuch, Band 1: Systematischer Teil, Darmstadt 2016, S. 305-308.

33 Die ältesten Stadtansichten stammen von Wilhelm Dilich; Paul Emil Richter (Hg.), Wilhelm Dilichs Federzeichnungen kursächsischer und meißnischer Ortschaften aus den Jahren 1626-1629 (Aus den Schriften der Königlich Sächsischen Kommission für Geschichte 13), 3 Bände, Dresden 1907, hier Band 1, Nr. 29-31.

34 Jacoв Grimm/Wilhelm Grimm, Deutsches Wörterbuch, Band 10, Leipzig 1877, Sp. 687.

35 Enno Bünz, Der Burg-Name in der Stadt. Beobachtungen vornehmlich in Mitteldeutschland, in: Dieter Kremer/Dietlind Kremer (Hg.), Die Stadt und ihre Namen, 2. Teilband: Akten (Onomastica Lipsiensia 9), Leipzig 2013, S. 11-26, hier S. 16-18. 
Lukas Cranachs d. Ä. Hofjagdbild von 1540 dargestellt, das Schloss Hartenfels zeigt (allerdings nicht mehr den Zustand, den Herzheimer gesehen hat), als Fialen hingegen auf dem Cranachbild „Das Goldene Zeitalter“ von ca. 1530, welches Schloss Hartenfels vor dem Umbau darstellen soll. ${ }^{36}$

Die Anlage des Schlosses bleibt im Einzelnen unklar, doch ist aufgrund der bekannten Baudaten von Schloss Hartenfels sicher, dass Herzheimer das Schloss noch in Bauformen gesehen hat, die wenige Jahrzehnte später durch den Neubau Johann Friedrichs d. Ä. beseitigt wurden. ${ }^{37}$ Rätselhaft bleibt dabei, was er mit der „fremden und neuen Art“ des Baus gemeint haben könnte; vielleicht fielen ihm Wendelstein und Vorhangbogenfenster als besonders fremdartig auf, die für den mitteldeutschen Schlossbau des ausgehenden 15. Jahrhunderts charakteristisch sind, ${ }^{38}$ aber in Altbayern unbekannt waren. Dass Herzheimer Renaissancearchitektur als Maßstab vor Augen stand, ist wenig wahrscheinlich, weil diese bis 1518/19 auch im Herzogtum Bayern noch nicht Fuß gefasst hatte. ${ }^{39}$

Bei aller Kürze der Schlossbeschreibung überliefert uns Herzheimer doch noch einige wenige Details der Innenausstattung. Er könne nicht alles beschreiben, bemerkt Herzheimer, yedoch noch ains khan ich nit onderlassen: In ir churf(urstlich) g(naden) slaff camere zw negst dem grossen schön vnd wolgeziertten furstlichem pedt ist an der ainen seytten gen der wondt, da mein herr vnd lieber vetter ligdt, ain cleynes schones stublen, das ist mit kraußin fladerem boltz alles ausgetafelt, ond stett dar inn ain schones tischlen (Editionsanhang Nr. 45). Für Wandverkleidungen mit gemasertem Holz ${ }^{40}$ hatte Friedrich der Weise auch sonst eine Vorliebe, wie Herzheimers Beschreibung des Wittenberger Schlosses zeigt. Auch dass es hier neben dem fürstlichen Schlafzimmer ein beheizbares An- und Auskleidezimmer gab, erscheint gar nicht so bemerkenswert. Interessant ist viel-

36 „Hofjagd auf Hirsche und Bären“ (1540) abgebildet in: MARx/KLUTH, Glaube und Macht, Katalog (wie Anm. 2), S. 227, Abb. 8; „Das Goldene Zeitalter“ (ca. 1530) abgebildet in: Stockhausen, Torgau (wie Anm. 1), S. 52, Abb. 4 (Ausschnitt). Für Auskünfte habe ich in diesem Zusammenhang Frau Dr. Anke Neugebauer (Halle/Wittenberg, Projekt: „Das ernestinische Wittenberg (1486-1547)“, Stiftung LEUCOREA) vielmals zu danken.

37 Findeisen/Magirius, Denkmale der Stadt Torgau (wie Anm. 1), S. 115-133; Steffen Delang, Schloss Hartenfels zu Torgau als Residenz, in: Stockhausen, Torgau (wie Anm. 1), S. 18-27.

38 Siehe die Beiträge in: Hendrik Bärnighausen (Bearb.), Schlossbau der Spätgotik in Mitteldeutschland, Dresden 2007.

39 Als Überblick Sigmund Benker, Die Kunstentwicklung vom sechzehnten bis zum Ende des achtzehnten Jahrhunderts, in: Max Spindler (Hg.), Handbuch der bayerischen Geschichte, Band 2: Das alte Bayern. Der Territorialstaat vom Ausgang des 12. Jahrhunderts bis zum Ausgang des 18. Jahrhunderts, München 21988, S. 1050-1117, hier S. 1051-1056 zur „Übernahme der antiken Formen“; BRIGITTE LANGER/KathaRINA Heinemann (Hg.), „Ewig blühe Bayerns Land“. Herzog Ludwig X. und die Renaissance, Regensburg/München 2009.

40 Gemasertes Holz wie z. B. Kiefer; „flader“ bezeichnet laut JАсов GRImm/Wilhelm Grimm, Deutsches Wörterbuch, Band 3, Leipzig 1862, Sp. 1708 f. speziell den Ahorn, aber auch allgemeiner geflecktes, geädertes, krauses Holz. 
mehr, dass Degenhard Pfeffinger als Kämmerer mit im großen Bett des Fürsten schlief: Vnd so obgemelter mein vetter ab dem furstlichem pedt her ab wil, so thutt er an der selben seitten ayn twelen [Vorhang] ${ }^{41}$ anf ond khumbt aus dem pedt in dasselb warmes ein gehaytztes schon schtubln ond thut sich dar inne an. Aus anderen Quellen scheint sich nichts über die Schlafgewohnheiten Kurfürst Friedrichs oder anderer Fürsten der Zeit zu ergeben, aber dass der Herr mit seinem Kämmerer das Bett teilte, ist doch recht bemerkenswert. ${ }^{42}$ Allerdings dürfte es die Regel gewesen sein, dass der Fürst sein Schlafgemach mit einem Teil des Gefolges teilte. Das älteste Wittenberger Schlossinventar von 1538/39 nennt in der kurfürstlichen Schlafkammer 1 betstadt, 1 schubbet, 3 underbeth, 2 oberbeth 2 strosecke, im Inventar von 1546 oder 1559 kommen zwei Bettstätten vor, und noch das Inventar von 1618 erwähnt neben dem fürstlichen Himmelbett ein Schubbett und ein Spannbett. ${ }^{43}$

Noch ein Detail der Schlosseinrichtung erwähnt der Reisebericht von 1519. Friedrich der Weise ließ aus mehreren hohen Fürstenzimmern ain zymer auf mein gnedigsten herrn, churf(urst) ond bischowe zw Mayntz etc., mit costlicher tapossterey auf richten. Allein der Fürstentisch sei mit sieben goldenen Teppichen bedeckt, und die Wände seien mit niederländischen gewirkten Seidenvorhängen (Tapisserien) verkleidet, die 600 und 800 Gulden gekostet hätten (Editionsanhang Nr. 46). ${ }^{44}$ Über den Zeitpunkt der Einrichtung des Fürstenzimmers kann ich noch keine Angaben machen. Wahrscheinlich geschah dies zu Ehren Albrechts von Brandenburg, der seit 1513 Erzbischof von Magdeburg und Administrator des Bistums Halberstadt war, seit 1514 aber auch noch das ranghöhere Erzbistum Mainz innehatte. ${ }^{45}$ Albrecht war der sächsische Kurfürst nicht nur reichspolitisch verbunden, sondern auch territorial, da ein Großteil des wettinischen Landes in Thüringen kirchlich zum Erzbistum Mainz gehörte, in Sachsen-Anhalt zudem

41 Bei Jacoв Grimm/Wilhelm Grimm, Deutsches Wörterbuch, Band 32, Leipzig 1954, Sp. 970-972 s. v. „Zwehle“; ebd., Band 22, Leipzig 1952, Sp. 1955 ist „twele“ als „Handtuch" nachgewiesen.

42 Friedrich der Weise war zwar nicht verheiratet, aber er hatte viele Jahre eine Lebensgefährtin, und aus dieser Verbindung gingen zwei illegitime Kinder, Fritz und Bastl, hervor; Ludolphy, Friedrich der Weise (wie Anm. 11), S. 47-53.

43 Freundliche Mitteilung von Frau Dr. Anke Neugebauer (Halle/Wittenberg) mit E-Mail vom 19. April 2013. Ihr verdanke ich auch den Hinweis auf ähnliche Verhältnisse in Schloss Hartenfels, die Stephan Hoppe aus den Inventaren des 17. Jahrhunderts erschlossen hat, siehe dazu Stephan Hoppe, Die funktionale und räumliche Struktur des frühen Schloßbaus in Mitteldeutschland. Untersucht an Beispielen landesherrlicher Bauten der Zeit zwischen 1470 und 1570 (Veröffentlichung der Abteilung Architektur des Kunsthistorischen Instituts der Universität Köln 62), Köln 1996, S. 152, 154.

44 Siehe allgemein Birgit Franke, Tapisserien, in: Werner Paravicini (Hg.), Höfe und Residenzen im spätmittelalterlichen Reich. Bilder und Begriffe, Teilband 1: Begriffe (Residenzenforschung 15.II/1), Ostfildern 2005, S. 90-92.

45 Friedhelm Jürgensmeier, Albrecht, Markgraf von Brandenburg, in: Erwin Gatz (Hg.), Die Bischöfe des Heiligen Römischen Reiches 1448 bis 1648. Ein biographisches Lexikon, Berlin 1996, S. 13-16. 
Teile des Territoriums kirchlich zum Erzbistum Magdeburg und Bistum Halberstadt gehörten. ${ }^{46}$

Schließlich geht Herzheimer noch auf die Schlosskirche ein, die sich aber noch nicht an der Stelle der heutigen Kapelle befand, die als einer der frühesten protestantischen Kirchenbauten zu einiger Berühmtheit gelangt ist. ${ }^{47}$ Die St. Martinskapelle lag 1519 vielmehr im Ostteil des Schlosses und wurde 1533 abgerissen. ${ }^{48}$ Die Kapelle war zweistöckig angelegt, also eine Doppelkapelle, was Herzheimers Angabe einer parkirche $=$ Emporenkirche verständlich macht. Die von ihm erwähnte Orgel ist schon 1462 nachweisbar. ${ }^{49}$ Nicht bekannt war hingegen, dass in der Kapelle vnser frawn tagzeitten taglich gesungen werden (Editionsanhang Nr. 47). Hierbei handelt es sich um eine besondere Form des Lobes der Muttergottes, nämlich die Abhaltung des Stundengebetes zu Ehren Mariens, wie es vielerorts in Mittel- und Norddeutschland im späten Mittelalter gestiftet wurde. Dafür hatten Kurfürst Friedrich und sein Bruder Johann am 7. Januar 1518 jährliche Einkünfte von 350 rheinischen Gulden gestiftet, um vier Priester, sieben Chorschüler, einen Organisten und drei Knaben zu finanzieren, ${ }^{50}$ - eine beachtliche Stiftung!

Auf die Residenzfunktion Torgaus muss ich im Rahmen dieses Beitrags schon deshalb nicht näher eingehen, weil erst vor kurzem Thomas Lang darüber publiziert hat. ${ }^{51}$ Die Erforschung der deutschen Fürstenhöfe und Residenzen des späten Mittelalters und der Frühen Neuzeit hat in den letzten 20 Jahren durch die Arbeit der Residenzen-Kommission bei der Göttinger Akademie der Wissenschaften erhebliche Fortschritte gemacht. Das 2003 erschienene Handbuch „Höfe und Residenzen im spätmittelalterlichen Reich" hat auf der einen Seite deutlich gemacht, wie zahlreich die Residenzorte auch in den mitteldeutschen Territorien waren, wie schlecht sie aber auch erforscht sind. Das gilt namentlich für die kursächsischen Residenzen. Auf die Frage, wo Kurfürst Friedrich der Weise residiert hat, würden historisch halbwegs Gebildete wohl antworten: in Wittenberg. Interessanterweise haben aber gerade die Forschungen, die in den letzten Jahren im Projekt „Ernestinisches Wittenberg 1486-1547“ durchgeführt wurden, zu dem überraschenden Ergebnis geführt, dass Wittenberg eben nur einer von mehreren Residenzorten war, an denen sich der Kurfürst aufhielt. ${ }^{52}$ Wittenberg war um 1500

46 Siehe die Bistumskarten in: Erwin Gatz (Hg.), Atlas zur Kirche in Geschichte und Gegenwart. Heiliges Römisches Reich, Deutschsprachige Länder, Regensburg 2009.

47 Hans-Joachim Krause, Die Schlosskapelle, in: Stockhausen, Torgau (wie Anm. 1), S. 38-47; Jürgen Herzog (Red.), Die Schlosskirche zu Torgau. Beiträge zum 450jährigen Jubiläum der Einweihung durch Martin Luther am 5. Oktober 1544, Torgau 1994. Findeisen/Magirius, Denkmale der Stadt Torgau (wie Anm. 1), S. 119 f.

49 Ebd.

50 Regest in: Carl Knabe (Hg.), Urkundenbuch von Torgau, Torgau 1902, S. 93, Nr. 230.

51 Thomas Lang, Zwischen Reisen und Residieren, in: Kohnle/Schirmer, Kurfürst Friedrich der Weise (wie Anm. 6), S. 201-229.

52 Heiner Lück u. a. (Hg.), Das ernestinische Wittenberg: Universität und Stadt (14861547) (Wittenberg-Forschungen 1), Petersberg 2011; LüCK, Das ernestinische Wittenberg: Stadt und Bewohner (wie Anm. 10); Heiner Lück u. a. (Hg.), Das ernestinische Wittenberg: Spuren Cranachs in Schloss und Stadt (Wittenberg-Forschungen 3), Petersberg 2015; weitere Bände sind in Vorbereitung. 
selbstverständlich als Zentrum der Kurlande von Bedeutung, mit deren Besitz die sächsische Kurwürde verbunden war, ${ }^{53}$ und verfügte mit dem Allerheiligenstift (die Schlosskirche) und der Landesuniversität über Institutionen, die in den anderen Residenzorten nicht vorhanden waren. Aber für Kurfürst Friedrich den Weisen spielten neben Wittenberg auch Torgau, Altenburg und Eilenburg, nicht minder aber die Jagdschlösser Lochau, das heutige Annaburg, und Colditz eine bedeutende Rolle, wobei auch festzustellen ist, dass die Jagdschlösser nicht Rückzugsorte des Fürsten waren, sondern als Bühnen für fürstliche Repräsentation dienten, beispielsweise wenn Gesandtschaften nach Kursachsen kamen. Die umfangreichen Rechnungsserien der kursächsischen Landesverwaltung geben über die Aufenthaltsorte und die Aufenthaltsdauer des Fürsten und seines Hofes umfassend und mit geradezu mathematischer Präzision Auskunft. Kurfürst Friedrich „besaß somit nicht eine Residenz, sondern mehrere Hoflager“. 54

Auch das Reisetagebuch Hans Herzheimers zeigt dies recht anschaulich, denn in den Monaten Januar bis Mai 1519, in denen Herzheimer durch Kursachsen reiste, hatte er eigentlich ständig den kurfürstlichen Hof im Blick und erwähnt vielfach die Aufenthaltsorte Friedrichs des Weisen. Allein vom 10. bis zum 17. Februar hat sich der Kurfürst laut Herzheimer trotz des winterlichen Wetters von Altenburg nach Zeitz begeben, von dort über Borna nach Grimma, dann nach Eilenburg, weiter nach Torgau und schließlich nach Wittenberg. Von Torgau nach Wittenberg reiste Friedrich der Weise entweder auf dem Landweg im Wagen oder im Schiff auf der Elbe. Wie Herzheimer erwähnt, hatte der Kurfürst in Torgau zwei große schyff mit hochen segln auf dem wasser steen ond sind rayn bedeckt ond gantz swartz an gestrichen. Ganz nebenbei weist unser Reisender an dieser Stelle auch auf die Bedeutung der Elbe als Handelsstraße hin: Daselb sicht man auch große schiff, die von Pehaym her ab komen mit trayd, wein vnd kaufmans guettern (Editionsanhang Nr. 52).55

Noch ehe Herzheimer aber das Residenzschloss beschreibt, wendet er sich dem Haus Degenhard Pfeffingers zu, das der Kurfürst seinem Kämmerer hatte neu errichten lassen (von grunndt newes erpawenn). Das Haus lag, wie Herzheimer angibt, gegenüber der Marienkirche, also in der Ritterstraße. ${ }^{56}$ Jürgen Herzog

53 Dazu Enno Bünz, Die sächsische Kurwürde - Geschichte und Bedeutung, in: Jutta Charlotte von Bloh/Dirk Syndram/Brigitte Streich (Hg.), Mit Schwert und Kreuz zur Kurfürstenmacht. Friedrich der Streitbare, Markgraf von Meißen und Kurfürst von Sachsen (1370-1428), München/Berlin 2007, S. 134-138; allgemeiner Ders., Kurwürde, in: Albrecht Cordes u. a. (Hg.), Handwörterbuch zur deutschen Rechtsgeschichte, Band 3, Berlin 22016, Sp. 365-370.

54 LANG, Zwischen Reisen und Residieren (wie Anm. 51), S. 229.

55 Vgl. hierzu Uwe Schirmer, Der Warenverkehr auf der Elbe zwischen Pirna und Wittenberg (1444-1545), in: Peter Rauscher/Andrea Serles (Hg.), Wiegen, Zählen, Registrieren. Handelsgeschichtliche Massenquellen und die Erforschung mitteleuropäischer Märkte (13.-18. Jahrhundert) (Beiträge zur Geschichte der Städte Mitteleuropas 25), Innsbruck u. a. 2015, S. 197-215.

56 Findeisen/Magirius, Denkmale der Stadt Torgau (wie Anm. 1), S. 404 als in dieser Straße liegend erwähnt, doch nicht genauer lokalisiert. 
hat ermittelt, dass es auf dem Grundstück Ritterstraße 10 lag, wo heute ein als Stadtbibliothek genutztes Barockpalais steht. Dem Torgauer Ratsprotokoll von 1525 zufolge wurde damals „das Priesterhaus des Lehens Steffani (heute Ritterstraße 8) zwischen des pfeffingers hauße (Ritterstraße 10) und Hans von Holden Haus (Ritterstraße 6) verkauft". ${ }^{57}$ Pfeffingers Haus befand sich in unmittelbarer Nähe von Kornhaus, Schösserei und Kanzlei, die hier gewissermaßen ein „Regierungsviertel“ zwischen Schloss und Marienkirche bildeten. ${ }^{58}$

Die Dimensionen und Einzelheiten des Pfeffingerschen Hauses waren bisher nicht bekannt. Es handelte sich dabei - ich zitiere Herzheimer - um ain schones zirrlichs hawss mit ainem hochen thurn, der vbr all hew $\beta$ auf geedt, von grundt newes erpawenn (Editionsanhang Nr. 43). Mit einiger Fantasie kann man den hochaufragenden Turm des Hauses sogar auf den Jagdbildern Lukas Cranachs d. Ä. von 1544 und 1545 ausmachen, die im Hintergrund die Stadtansicht Torgaus von Osten zeigen.59 Angesichts der Dimensionen des Gebäudes fasst sich Herzheimer kurz, denn man könne lange davon schreiben, wie es erpauen ist mit vil schonen stuben, cammern, lussthewseren vnd vil gemachen, hatt mer dann 400 tur, auch ain lustige schone wolgezierte cappellen, padhaws, prunnen, gartten ond stallen. Das Inventar des Hauses benennt Herzheimer ebenfalls nur summarisch, verweist auf den haußratt in den chuchellen, padgeschirren, silbergeschirren, petgewandt, zyngeschirren, alles aufs raynest aus erfullt, an nichts bestünde Mangel. Auch Kräutergärten (wurtzgertten) und anderes mehr erwähnt er: Wer es alles sollte beschreiben, der muest die gemache all von grundt vnden anfachen ond bis vber das dach auf volenden. Das aber hat unserer bayerischer Reisender leider nicht getan, obwohl er - wie an späterer Stelle seines Reiseberichts zu erfahren ist - im April und Mai 1519 wegen seines kranken Beins mehrere Wochen in herrn Degnharts hauss beliben sei. Auch wenn die Angaben, in Pfeffingers Haus habe es

57 Briefliche Mitteilung von Herrn Dr. Jürgen Herzog vom 21. Januar 2015 nach Stadtarchiv Torgau, HZ60a, Ratsprotokolle 1525, fol. 27r-v. Im selben Jahr wurde auch (ebd., fol. 13r) das Haus des Erzpriesters Paul Nyman gegen Pfeffinger haus uber gelegen verkauft. Die Lage schräg gegenüber der Stadtpfarrkirche ergibt sich laut Dr. Herzog auch nach KarL-Heinz Lange, Häuserbuch der Stadt Torgau, Leipzig 2013, S. 326. Wie Ausgaben für Baumaßnahmen an Pfeffingers Haws Gothseligen 1523 belegen (Thüringisches Hauptstaatsarchiv, EGA, Reg. Cc 1182, Geleitsrechnung 1523, fol. $113 \mathrm{v}-114 \mathrm{v})$, befand sich das Gebäude nach seinem Tod in landesherrlichem Besitz, doch muss es bereits 1531 in Stadtrecht gekommen sein, weil es damals Merten Proschwitz besaß; siehe LANGE, Häuserbuch (a. a. O.), S. 328 (dort aber z. T. falsche Angaben). Der Begriff „Regierungsviertel“ bei Butz, Torgau (wie Anm. 2), S. 584. Zum Kanzleihaus siehe die Beiträge in: BAIER, Das kurfürstliche Kanzleihaus (wie Anm. 1).

59 Das Jagdbild von 1544 (Museo del Prato, Madrid) abgebildet in Stockhausen, Torgau (wie Anm. 1), S. 50; das von 1545 (Museo del Prato, Madrid) abgebildet ebd., S. 48. Auf beiden Bildern ist zwischen dem Schloss und der Marienkirche ein hoher Turm hinter der Stadtmauer zu erkennen. Weniger eindeutig ist die Darstellung auf dem Jagdbild Lukas Cranachs d. J. von 1544 (Kunsthistorisches Museum, Wien), das zwischen Schloss und Marienkirche einen Turm in der Stadtmauer zeigt, der auf den eben genannten Bildern nicht zu sehen ist, rechts davon hinter der Stadtmauer aber ebenfalls ein höheres Gebäude oder einen Turm zeigt. 
400 Türe gegeben, arg übertrieben erscheint, ist doch nicht von der Hand zu weisen, dass es sich um ein stattliches Wohnhaus mit Badehaus, Turm und eigener Kapelle, Gartenanlagen und reichhaltigem Hausrat gehandelt haben muss. ${ }^{60}$ Aus der Torgauer Geleitsrechnung von 1523 ist zu entnehmen, dass damals umfangreiche Bau- und Sanierungsarbeiten durchgeführt wurden: so wurde das Bleidach auf den beiden Gängen am Turm abgedeckt, ebenso die Turmbedachung. Auch das Dach am obersten Gang zur Kapelle und das Kapellendach selbst wurden wieder eingedeckt. Der Gang im Hof neben der Kapelle wurde ausgebessert und der Röhrkasten (rorekasten) erneuert. ${ }^{61}$

Als Pfeffinger im Mai 1519 mit seinem Vetter Herzheimer Torgau verließ, um zur Kaiserwahl nach Frankfurt am Main zu reisen, konnte er nicht ahnen, dass er nie wieder zurückkehren würde. Am 3. Juli 1519 ist Pfeffinger in Frankfurt an einer grassierenden Seuche gestorben. ${ }^{62}$ Wie Hans Herzheimer berichtet, wurde Pfeffingers Leichnam in der dortigen Franziskanerkirche beigesetzt (an ihrer Stelle steht heute die sogenannte Paulskirche), und Kurfürst Friedrich der Weise habe nicht nur Stiftungen für das Seelenheil seines Kämmerers vorgenommen, sondern auch dafür Sorge getragen, einen Totenschild anbringen und einen Grabstein hauen zu lassen. Dies verdeutlicht noch einmal die besondere Verbundenheit des Kurfürsten mit seinem Kämmerer. Das Grabmal am Stammsitz Degenhard Pfeffingers in Salmanskirchen, das ihn im prächtigen Maximiliansharnisch zeigt, ist also ein bloßes Kenotaph, ein Erinnerungsmal. ${ }^{63}$

Von der Umgebung des Pfeffinger-Hauses erwähnt Herzheimer leider nur, dass auf der Straßenseite gegenüber die Marienkirche (vnnser frawen cirichen) liegt, die fast wol erpawen, doch lässt er sich über die Baugestalt und Ausstattung der spätgotischen Hallenkirche leider nicht weiter aus. ${ }^{64} \mathrm{Hier}$ wie an vielen anderen Stellen wird deutlich, dass Herzheimer auf kirchliche Dinge nur ganz kursorisch eingeht, sei es nun, weil es ihn nicht sonderlich interessierte, oder weil er es einfach nicht für wichtig hielt, die Kirchen näher zu beschreiben (bei der Wittenberger Schlosskirche und der neuen Pfarrkirche von Annaberg im Erzgebirge hat er es dann aber doch anders gehalten und weiter ausgeholt) ${ }^{65}$. Alles, was mit dem

60 Zur Innenausstattung Torgauer Bürgerhäuser der Zeit siehe Angelica Dülberg, Wand- und Deckenmalereien in Torgauer Bürgerhäusern und im Schloss Hartenfels, in: Stockhausen, Torgau (wie Anm. 1), S. 122-135.

61 Thüringisches Hauptstaatsarchiv, EGA, Reg. Cc 1182, Geleitsrechnung 1523, fol. $113 v-114 v$ (für die Mitteilung der Quellenauszüge habe ich Herrn Dr. Jürgen Herzog vielmals zu danken). - Im Röhrkasten sammelte sich das Wasser aus der Röhrleitung; siehe zum Begriff Jaсов Grimm/Wilhelm Grimm, Deutsches Wörterbuch, Band 14, Leipzig 1893, Sp. 1132; zur Torgauer Röhrwasserleitung Findeisen/Magirius, Denkmale der Stadt Torgau (wie Anm. 1), S. 90.

62 Dazu Bünz, Wettiner auf den Reichstagen (wie Anm. 13), S. 450 f.

63 BüNZ, Heiltumssammlung (wie Anm. 16), S. 159-162.

64 Findeisen/Magirius, Denkmale der Stadt Torgau (wie Anm. 1), S. 241-312; Heinrich Magirius, Marienkirche, in: Stockhausen, Torgau (wie Anm. 1), S. 58-69; Ders., Marienkirche Torgau (Schnell Kunstführer 1995), Regensburg ${ }^{3} 2007$.

65 BüNZ, Wittenberg 1519 (wie Anm. 10), S. 20-22. 
Fürstenhaus zu tun hat, interessierte ihn viel mehr, und deshalb hebt er bezüglich der Torgauer Marienkirche auch als einziges Ausstattungselement hervor, dort läge meins g(nedigen) herrn herzogs Johans von Sachsen etc. elich gemachel fraw Sophia, ain fursstin der gepurdt aus dem fursstenthumb Mecklburg etc. begraben. Herzogin Sophie von Mecklenburg war 1503 verstorben. Das Grab befand sich vor dem Annenaltar im Hauptchor und wurde erst 1696 an die heutige Stelle im südlichen Nebenchor verlegt, und dort befindet sich noch die 1504 angefertigte Bronzegrabplatte der Vischer-Werkstatt aus Nürnberg. ${ }^{66}$

Hätte Herzheimer in Torgau mehr Zeit gehabt, wäre ihm in der Marienkirche vielleicht noch manches andere aufgefallen, etwa der „Stuhl auf der Empore für Herrn Pfeffingers Frau“, der 1514 errichtet werden sollte, ${ }^{67}$ die von den Landesherren gestifteten Altäre und die Fürstenempore. Hierzu gehört aber ein interessantes Detail, das unser Reisender bereits im Zusammenhang mit dem Schloss erwähnt hat: Aus dem sloss baben ir churf(urstlich) g(naden) ain(en) kunstlichen ganck bis in die vor ermelt vnnser frawen kirichen gefuerdt, furtter kbumbt der selb gangk aus vber die stattmawer ond statt graben, vber die landstrass, in ain(en) schenen lusstigen pawmgarten (Editionsanhang Nr. 44). Der gedeckte Verbindungsgang lief folglich vom Schloss zum Nordchor, wo sich noch ein repräsentatives Eingangsportal erhalten hat. ${ }^{68}$ Die Stadtansicht Wilhelm Dilichs von 1628 und der Merian-Stich von 1650 lassen den Verbindungsgang noch gut erkennen. ${ }^{69}$

Eigentlich spektakulär an Herzheimers Bericht ist aber die Beschreibung der Umgebung des Schlosses, vor allem des Gartens mit seinen Bauten, wobei bemerkenswert ist, dass der erwähnte gedeckte Gang vom Schloss zur Marienkirche, von dort über die Stadtmauer weiter in diesen Baumgarten führte, der nördlich der heutigen Elbstraße am Ufer der Elbe lag, ${ }^{70}$ und - wie dem Inventar von $1601 \mathrm{zu}$

66 Findeisen/Magirius, Denkmale der Stadt Torgau (wie Anm. 1), S. 291 und Abb. 269; Sven Hauschke, Die Grabdenkmäler der Nürnberger Vischer-Werkstatt (1453-1544) (Denkmäler Deutscher Kunst. Bronzegeräte des Mittelalters 6), Berlin u. a. 2006, S. 186189, Nr. 16 mit Abb. 133-134. - Das Grabensemble behandeln die Katalogartikel in: Hartmut Kühne/Enno Bünz/Thomas T. Müller (Hg.), Alltag und Frömmigkeit am Vorabend der Reformation in Mitteldeutschland, Katalog zur Ausstellung „Umsonst ist der Tod“, Petersberg 2013, S. 101-106. - Jürgen Herzog, Das Grabmal der Herzogin Sophie von Sachsen in der Stadtkirche St. Marien in Torgau, in: Torgauer Heimatkalender 8 (2013), S. 32-35. - Ders., Fürstlicher Hof (wie Anm. 6), S. 297-303.

67 Findeisen/Magirius, Denkmale der Stadt Torgau (wie Anm. 1), S. 248.

68 Ebd., S. 248 mit Abb. 230 und S. 195.

69 Beide Stadtansichten mehrfach abgebildet, z. B. von Armin Schneiderheinze, Das Inventarium von 1601. Beobachtungen zu Struktur, Funktionsweise und Ausstattung der Kurfürstlichen Kanzlei und des Torgauer Amtes, in: Baier, Das kurfürstliche Kanzleihaus (wie Anm. 1), S. 82-124, hier S. 82 f., S. 85 und vergrößerte Ausschnitte S. 86 f. Zum Kirchgang auch ebd., S. 93 f.

70 Zur Lokalisierung Findeisen/Magirius, Denkmale der Stadt Torgau (wie Anm. 1), S. 201, wo die Elbstraße noch als Straße der Deutsch-Sowjetischen Freundschaft bezeichnet wird. Zur Lage auch Herzheimer: Vnd neben dem selben gartten fleusst ain gross schiffreiches wasser fur, genandt die Ellwen, fleust aus dem land zw Pebaym (Editionsanhang Nr. 45). 
entnehmen ist - im dritten Geschoss der unten beschriebenen schießhutten endete: ${ }^{71}$ In dem selben gartten baben ir churf(urstlich) g(naden) gepawen zbay schon lussthere $\beta$ mit raynen lusstigen gemachen ond aufs kunsstlichest aus malen lassen. Von diesen Sommerhäusern ist nichts erhalten geblieben, weil dieser Bereich im Dreißigjährigen Krieg 1637 zerstört wurde. ${ }^{72}$ Lediglich das Torgauer Amtsinventar von 1601 verzeichnet noch die von Herzheimer erwähnten Gebäude, nämlich das Schießhaus und zwei flankierende Lusthäuser. ${ }^{73}$ Im Baumgarten befand sich auch ein sogenanntes Wildbad ${ }^{74}$ mit einer natürlichen Quelle: Auch dar inne ain lusstiges wildpald, die selbig wasserstuben im wildpald ist alle mit zyn aus gemacht, ond ain lusstige padstuben dabei, vnd was von padgeschier dar ein gehordt, das ist alles von khuppfaren geschieren inwendig rayn ausgezynedt..$^{75}$ Da ist kayn geprechen noch mangl, ond sol man ewig leben. Gewiss hat Friedrich der Weise dieses Thermalbad genutzt, denn er litt in seinen späten Jahren an der Gicht und hatte ein Steinleiden, wie wir aus zahlreichen Zeugnissen wissen. ${ }^{76}$

Von den Einrichtungen im Torgauer Baumgarten erwähnt Herzheimer noch eine kunstvoll angelegte Vogeltenne (im gartten ain khunstlicher vogel thenne, also ein Vogelherd, zw dem vinckenfanck gemacht). ${ }^{77}$ Als weiteres Gebäude nennt er ain lusstige schießhutten, so man zum zyl schiessen wil,78 und des Weiteren ain lusstige trinckstuben sowie noch mer lustige gepew zum ausseben. In ihnen nahmen also Gäste Platz, um den diversen Freizeitvergnügungen beiwohnen zu können, so man scheusst, oder wan man daselb auch rennedt oder sticht, ${ }^{79}$ das alles

71 SChNeIderheinze, Inventarium (wie Anm. 69), S. 114.

72 Kurz zu dieser Beschreibung Ludolphy, Friedrich der Weise (wie Anm. 11), S. 126; ausführlicher in Findeisen/Magirius, Denkmale der Stadt Torgau (wie Anm. 1), S. $201 \mathrm{f}$. - Der Baumgarten ist auch auf der Torgauer Stadtansicht Wilhelm Dilichs von 1628/29 erkennbar; siehe Dilich, Federzeichnungen I (wie Anm. 33), Nr. 29, im Vordergrund des Bildes mit dem Buchstaben n bezeichnet.

73 Schneiderheinze, Inventarium (wie Anm. 69), S. 88 f. und Edition S. 113 ff. Das Schießhaus auf der Stadtansicht von 1628/29 (vgl. Anm. 72) mit dem Buchstaben y bezeichnet.

74 Zum Begriff Jасов Grimm/Wilhelm Grimm, Deutsches Wörterbuch, Band 30, Leipzig 1960, Sp. 39.

75 Dies ist wohl das im Amtsinventar von 1601 beschriebene Gärtnerhaus; ScHNEIDERHEINZE, Inventarium (wie Anm. 69), S. 88 und S. 119.

76 Ludolphy, Friedrich der Weise (wie Anm. 11), S. 58-60.

77 Zum Begriff Jасов und Wilhelm Grimm, Deutsches Wörterbuch, Band 26, Leipzig 1951, Sp. 426 f. und ebd. Sp. 412 s. v. Vogelherd; S. Schwenk, Vogelherd, in: Lexikon des Mittelalters, Band 8, München 1997, Sp. 1810. - Diese Vorrichtung wird im Amtsinventar von 1601 nicht mehr genannt.

78 Im Amtsinventar von 1601 als Schißhaus; SCHNEIDERHEInZe, Inventarium (wie Anm. 69), S. 113 f. Dieses umfasste 68 Schießstände auf drei Etagen, darunter vier Schießstände im Frauenzimmer; ebd., S. 88.

79 Die Rennebahn, ein umzäunter Turnierplatz mit einem Tor, über dem das kursächsische Wappen angebracht war, wird im Inventar von 1601 beschrieben; SCHNEIDERHEINZE, Inventarium (wie Anm. 69), S. 115. Sie ist auf der Stadtansicht von 1628/29 rechts im Vordergrund dargestellt, bezeichnet mit dem Buchstaben z, siehe Dilich, Federzeichnungen I (wie Anm. 33), Nr. 29. 
neben der zylstatt bschehen khan, das mag man alles aus dem selben lussthauss, frawen vnd junckfr(awen), sehen, vnd nichts weniger, khartten, singen, essen oder trincken, oder saytenspill horen, als wär man im paradies ${ }^{80}$ (Editionsanhang $\mathrm{Nr}$. 45). Hans Herzheimer scheint sich in Torgau wohlgefühlt zu haben.

Dazu mag auch beigetragen haben, dass er dort im Mai 1519 - die kommende Kaiserwahl warf ihre Schatten voraus - dem Besuch einer großen Gesandtschaft beiwohnen konnte, die der Habsburger König Karl von Spanien zu Kurfürst Friedrich geschickt hatte, um ihn für seine Wahl zum Kaiser des Heiligen Römischen Reiches zu gewinnen. Die 200-köpfige Gesandtschaft, der u. a. Markgraf Kasimir von Brandenburg und Herzog Otto von Braunschweig-Lüneburg sowie zahlreiche Grafen und Herren angehörten, traf am 11. Mai - aus Schweinitz kommend, wo sie an einem birschgejaid teilgenommen hatte - in Torgau ein und verlustierte sich im beschriebenen Baumgarten an der Elbe: Ampfintztag, das ist der 12. Mai gewesen, mit der potschafft das fruemal im pawmgartten gehalten ond rennen lassen. Es wurde also im Baumgarten ein Turnier veranstaltet. Danach wurde die Gesandtschaft dann verabschiedet, indem ihr noch das Geleit bis zur Heilig-Kreuz-Kapelle vor dem Spitaltor gewährt wurde (Editionsanhang Nr. 195). Die Gesandtschaft mag ihren Zweck erfüllt haben, denn Kurfürst Friedrich von Sachsen gehörte am 28. Juni 1519 zu den Wählern Karls V.

Neben diesem Baumgarten mit seinen Sommerhäusern erwähnt Herzheimer noch etliche weitere Bauten im Umfeld des Schlosses. Da ist zunächst das Zeughaus, das sich schon damals an der heutigen Stelle befand und eine Doppelfunktion als Getreidespeicher und Waffenlager hatte: Es haben ir churf(urstlich) g(naden) auch bey dem sloss ainen grossenn casten mit getraydt oben lygen, ond vnden mit grossem geschutz, haubttpuchssen, morthen, slänglen ond menigerlay veldgeschutz (Editionsanhang Nr. 48). 81

Vor dem sloss im velde hatte der Kurfürst laut Herzheimer einen großen Teich anlegen lassen, um Karpfen zu züchten. Höchstwahrscheinlich ist damit das Gewässer südlich der Stadt gemeint, das noch heute Großer Teich heißt. Überschlägig beziffert Herzheimer den Ertrag auf 3500 Gulden, doch wanderten etliche Karpfen auf den Tisch des Hofes (Editionsanhang Nr. 49). Wenn tatsächlich der Große Teich gemeint ist, trog Herzheimer allerdings die Erinnerung, da er im unmittelbaren Anschluss von der Heilig-Kreuz-Kapelle schreibt, die er in dessen Nähe lokalisiert. Diese Kapelle lag aber im Gebiet vor dem Spitaltor am Weg nach Leipzig, also nicht südlich, sondern nordwestlich der Stadt, ${ }^{82}$ wie dem Bericht Herzheimers zu entnehmen ist: Am 12. Mai 1519 erhielt eine Gesandtschaft von

80 Er setzt noch hinzu: Also ist das selbig lussthaus inwendig mit schoner tappesterei und kunstlichen gemall auß beraytt (Editionsanhang Nr. 45). Auf welches Gebäude dies zu beziehen ist, bleibt unklar.

81 Zum Korn- und Zeughaus (heute Schlossstraße 26) Findeisen/Magirius, Denkmale der Stadt Torgau (wie Anm. 1), S. 83 f.; und Stockhausen, Torgau (wie Anm. 1), S. 173.

82 Siehe Jürgen Herzog, Die Wallfahrtskapelle zum Heiligen Kreuz vor Torgau, in: Torgauer Heimatkalender 9 (2014), S. 29-33, hier S. 31 mit Eintragung im Stadtplan von 1810; Herzog, Fürstlicher Hof (wie Anm. 6), S. 305-307. 
Torgau aus das gelait anf dem wagen bis zum heiligen crentz, liess sy den tag gen Leibtzigk 6 meyl wider an ir gewär zw iren pferden farenn (Editionsanhang Nr. 195). Die Heilig-Kreuz-Kapelle hatte Kurfürst Friedrich der Weise vor seiner Pilgerreise nach Jerusalem gestiftet und nach seiner Rückkehr 1494 errichtet. ${ }^{83} 1499$ ist die Kapelle durch Erzbischof Ernst von Magdeburg geweiht worden. ${ }^{84}$ Bei dieser kleinen, aber reich verzierten Kirche geschähen - so Herzheimer - große wunder zaichen, der man vil in der selben kirch sieht. Das deutet wohl auf Votivgaben, die Pilger bei Gebetserhörung in der Kapelle hinterlassen haben. Die älteste Bilddarstellung des Bennograbes im Dom zu Meißen aus dem frühen 16. Jahrhundert zeigt noch eine solche Pilgerstätte mit den aus Wachs gefertigten Votivgaben, die Gläubige am Grab Bennos aufgehängt haben. ${ }^{85}$ Gar nicht ungewöhnlich ist auch die Ursprungslegende der Torgauer Wallfahrtskapelle, erwähnt Herzheimer doch, dass sich vor der kirichen in aynem grossen pawin ain gross crucifix befände; es lasse sich, so lesen wir, nicht aus dem Baum entfernen, denn es komme, egal wohin man es trage, wieder in den Baum zurück. Solche Entstehungslegenden sind nicht ungewöhnlich, wie beispielsweise die Anfänge der Wallfahrt zum heiligen Birnbaum in Rötha bei Leipzig, in Elende bei Nordhausen oder in Grimmenthal bei Meiningen in Südthüringen zeigen. ${ }^{86}$ Ein neueres Inventar der mittelalterlichen Wallfahrtsorte in Sachsen weist im „Mutterland der Reformation“ mit mehr oder minder plausiblen Argumenten über 70 Gnadenstätten nach. Die Heilig-Kreuz-Kapelle hier in Torgau findet sich dort aber ebenso wenig verzeichnet wie vnnser frawen kirichen im Voglgesanck (Editionsanhang Nr. 193), gemeint ist die Marienkirche in Vogelgesang, etwa $6 \mathrm{~km}$ nordwestlich von Torgau, die Herzheimer bei seinem zweiten Besuch in Torgau am 27. April 1519 aufgesucht hat. Auch dort bestand vor der Reformation eine der vielen Lokalwallfahrten, die nur wenige Spuren in den Quellen hinterlassen haben.87

Angesichts des Zusammenhangs der Torgauer Kapellenstiftung mit der Wallfahrt Kurfürst Friedrichs des Weisen ins Heilige Land überrascht es nicht, dass sich neben der Heilig-Kreuz-Kapelle ein Heiliges Grab befand. Herzheimer schreibt: Es hat ir churf(urstlich) g(naden) auf dem selben kirichboff ain geleichnuss des heiligen grabes, wie man es zw Jerusalem hatt, auf machen lassen (Editionsanhang Nr. 51). Diese Nachbildung des Heiligen Grabes war offenkundig ein Reflex der Pilgerreise Friedrichs des Weisen nach Jerusalem 1493. Daran erinnert

83 Findeisen/Magirius, Denkmale der Stadt Torgau (wie Anm. 1), S. 338 f.

84 Ludolphy, Friedrich der Weise (wie Anm. 11), S. 128.

85 Abgebildet in: KüHNE/Bünz/Müller, Alltag und Frömmigkeit (wie Anm. 66), S. 148.

86 Birgit Franke, Mittelalterliche Wallfahrt in Sachsen. Ein Arbeitsbericht, in: Arbeitsund Forschungsberichte zur sächsischen Bodendenkmalpflege 44 (2002), S. 299-389; Gabriela Signori (Hg.), Das Wunderbuch Unserer Lieben Frau im thüringischen Elende (1419-1517) (Veröffentlichungen der Historischen Kommission für Thüringen, Große Reihe 12), Köln u. a. 2006; Johannes Mötsch (Hg.), Die Wallfahrt zu Grimmenthal. Urkunden, Rechnungen, Mirakelbuch (Veröffentlichungen der Historischen Kommission für Thüringen, Große Reihe 10), Köln u. a. 2004.

87 Einige Nachweise bei Herzog, Fürstlicher Hof (wie Anm. 6), S. 309 f. 
heute noch ein großformatiges Tafelgemälde, das sich im Schlossmuseum Gotha befindet und 1522 nach einer Vorlage aus der Wittenberger Schlosskirche kopiert wurde. Das Bild zeigt in dichter Komposition die wichtigsten Wallfahrtsziele im Heiligen Land: In der Bildmitte links die Stadt Jerusalem, in der durch den kreuztragenden Christus die Via Dolorosa angedeutet ist, dahinter hochaufragend der heilige Berg Tabor, im Bildhintergrund rechts der Jordan, davor Bethlehem, die Geburtsstadt des Herrn. Im Vordergrund kniet in Gebetshaltung Kurfürst Friedrich der Weise von Sachsen, durch eine Inschrift bezeichnet, der zudem unschwer an seiner Physiognomie, aber auch dem vor ihm aufgebrachten Wappen erkennbar ist. Das Schiff rechts im Bild verweist auf den Reiseweg, den der Kurfürst und seine 188 Mitreisenden genommen hatten. Die Pilgerreise von 1493 war die aufwendigste wettinische Wallfahrt des späten Mittelalters überhaupt. ${ }^{88}$ Auf dieser Pilgerreise hat übrigens der Kurfürst den niederbayerischen Adligen Degenhard Pfeffinger kennengelernt und danach an seinen Hof geholt. ${ }^{89}$ Wäre das nicht geschehen, wäre Hans Herzheimer 1518/19 wohl nicht durch Sachsen gereist.

Von der Pilgerfahrt ins Heilige Land 1493 brachte Friedrich der Weise nicht nur zahlreiche Reliquien mit, die schließlich ins Wittenberger Heiltum gelangt sind, sondern er hat auch eine Nachbildung des Heiligen Grabes errichten lassen. Die Torgauer Anlage ist zwar spurlos verschwunden, ${ }^{90}$ sie dürfte aber dem Nachbau des Heiligen Grabes geglichen haben, der seit 1480 im Laufe von gut zwei Jahrzehnten in Görlitz im Auftrag des Stadtrates errichtet wurde. ${ }^{91}$ Das dortige Heilige Grab gleicht in vielen Einzelheiten dem Vorbild der Jerusalemer Grabeskirche. Kreuzkapelle und Grabkirche in Torgau waren von einer Mauer umgeben, was dafür spricht, das die Anlage der in Görlitz ähnelte. ${ }^{92}$ Ein solcher Nachbau war natürlich kein bloßes Reiseandenken, sondern verdeutlicht die Frömmigkeitspraxis des ausgehenden Mittelalters, die christuszentriert war und darauf zielte, die Leidensgeschichte des Herrn möglichst anschaulich nachvollziehbar zu machen. Nachbauten wie das Heilige Grab, aber auch zahlreiche Reliquien aus dem

88 Ludolphy, Friedrich der Weise (wie Anm. 11), S. 351-354; Martin StadeczeK, Rechnungsbuch Friedrichs des Weisen über die Reise ins Heilige Land, in: Kühne/Bünz/ Müller, Alltag und Frömmigkeit (wie Anm. 66), S. 173 f.

89 BüNZ, Heiltumssammlung (wie Anm. 16), S. 134 f.

90 Siehe nun ausführlicher Herzog, Wallfahrtskapelle (wie Anm. 82), S. 29-33. - In Findeisen/Magirius, Denkmale der Stadt Torgau (wie Anm. 1), S. 338 f. findet sich im Zusammenhang mit der Hl. Kreuz-Kapelle, aber auch an anderer Stelle kein Hinweis auf das Heilige Grab.

91 Ines Anders/Marius Winzeler (Hg.), Lausitzer Jerusalem. 500 Jahre Heiliges Grab zu Görlitz (Schriftenreihe der Städtischen Sammlungen für Geschichte und Kultur (Görlitz) N. F. 38), Görlitz/Zittau 2005; ERnst-Heinz Lemper, Görlitz. Heiliges Grab mit Kreuzkapelle (Schnell Kunstführer 2017), Regensburg 22004.

92 Dies betont auch Ludolphy, Friedrich der Weise (wie Anm. 11), S. 128. - Zur Frage der Ähnlichkeit solcher Heilig-Grab-Nachbildungen siehe aber ARnOld EscH, Anschauung und Begriff. Die Bewältigung fremder Wirklichkeit durch den Vergleich in Reiseberichten des späten Mittelalters, in: Ders., Zeitalter und Menschenalter. Der Historiker und die Erfahrung vergangener Gegenwart, München 1994, S. 70-92, hier S. 83-86. 
Heiligen Land, die an das Heilsgeschehen erinnerten, dienten als Hilfsmittel zu frommer Andacht. Bereits Ingetraut Ludolphy hat in ihrer Biografie Friedrichs des Weisen darauf hingewiesen, dass zur Heilig-Kreuz-Kapelle, die vor der Stadt lag, die Karfreitagsprozession führte. ${ }^{93}$ Womöglich entsprach auch die Entfernung präzise der Via Dolorosa, dem Leidensweg des Herrn in Jerusalem. 1533, schon wenige Jahre nach Einführung der Reformation, sind Kapelle und Heiliges Grab abgebrochen worden. ${ }^{94}$

Natürlich ist Hans Herzheimer auch über die berühmte Elbbrücke geritten, die Friedrich der Weise in Torgau recht langwierig von 1494 (Grundsteinlegung) bis 1517 errichten ließ: ${ }^{5}$ Es haben ir curf(urstlich) g(naden) vber das gross wasser, die Elben, ain kunstliche prucken mit gemawerten wasserstuben ond jochern pawen lassen, als man mich bericht, das die vber 12000 gulden zepawen gestanden hab (Editionsanhang Nr. 52). Allerdings dürfen wir hier Herzheimer nicht ganz trauen, denn nur die ersten drei stadtseitigen Pfeiler der Brücke wurden aus Stein errichtet. Dann führte man den Brückenbau nach jahrelanger Unterbrechung als Holzkonstruktion weiter. In dieser Gestalt wird die Brücke auch auf den Jagdbildern Lukas Cranachs d. Ä. 1544/45 dargestellt. Sie ist im Dreißigjährigen Krieg 1637 niedergebrannt worden. Interessant ist natürlich Herzheimers Angabe der enormen Bausumme von 12000 Gulden. Sein Vetter, der kursächsische Kämmerer Pfeffinger, mag ihm die Summe genannt haben. Dass Friedrich der Weise einen erheblichen Teil der Baukosten finanzierte, ist bekannt. Ebenso wissen wir, dass der Kurfürst seit 1491 für mehrere Jahrzehnte päpstliche Fastendispense (keine Ablässe, wie immer wieder zu lesen ist) nutzte, um den Brückenbau zu finanzieren. Gegen eine bestimmte Geldsumme konnten die Gläubigen im Kurfürstentum eine Dispens erwerben, die es ihnen gestattete, auch in den Fastenzeiten Milchprodukte und Butter zu verzehren. ${ }^{96}$ Brückenbauten galten im Mittelalter als frommes Werk, weshalb die Baufinanzierung auf diese Weise durch die Amtskirche gefördert wurde. ${ }^{97}$

93 Ludolphy, Friedrich der Weise (wie Anm. 11), S. 128.

94 Ebd.

95 Ebd., S. 126 f. - Findeisen/Magirius, Denkmale der Stadt Torgau (wie Anm. 1), S. 91 95. - Herzog, Fürstlicher Hof (wie Anm. 6), S. 289 f. - Auf den oben in Anm. 59 nachgewiesenen Stadtansichten Cranachs ist die Elbbrücke stets dargestellt.

96 H. Zapp, Butterbriefe, in: Lexikon des Mittelalters, Band 2, München/Zürich 1983, Sp. 1162 f.; Matthias Klipsch, Vom Fasten bei Wasser und Brot bis zum Fleisch- und Buttergenuss. Spätmittelalterliche Buß- und Fastenpraxis im Spiegel kurialer Registerüberlieferung, in: Michael Matheus (Hg.), Friedensnobelpreis und historische Grundlagenforschung. Ludwig Quidde und die Erschließung der kurialen Registerüberlieferung (Bibliothek des Deutschen Historischen Instituts in Rom 124), Berlin u. a. 2012, S. 279-302.

97 Nikolaus Paulus, Geschichte des Ablasses am Ausgang des Mittelalters, Darmstadt 2000 (Nachdruck der Ausgabe Paderborn 1923), S. 370-374 zu den zahlreichen Brückenablässen, hier S. 372 f. auch Angaben zur Torgauer Fastendispens, nach der jeder Gläubige, der davon Gebrauch machte, 1/20 eines Guldens für den Brückenbau zahlte. 
Indem wir mit Hans Herzheimer die Elbbrücke überschreiten, nehmen wir Abschied von Torgau. Als letzte Station beschreibt der bayerische Adlige jenseits der Elbe ain schones holtz genant die Strudt, dar auff ist nie anderes wildpratt dann eytl hirschen gefunden (Editionsanhang Nr. 53). Es handelt sich bei diesem Wald wohl um den südlichen Ausläufer der Annaburger Heide, der heutigen Lochauer Heide, denn Herzheimer erwähnt, dass man durch das holtz an das Vngrisch land gen Toberlaw wnd gen Hertzberig komme: Doberlug und Herzberg in der Niederlausitz unterstanden damals König Ludwig von Böhmen und Ungarn, deshalb „ungarisches Land“. 98 Dieser Wald war ein besonderes Jagdgebiet des Kurfürsten, wie Herzheimer hervorhebt, denn dort stendt die bynnd ond wild ond byrsch bey ein ander, weshalb der Kurfürst dieses Jagdgebiet besonderen Gäste vorbehalte: dan so man daselb auf der Strud jagt, so lauffn 20 oder mer hirsch auf ain mal herfur, ond ist so nachent von dem scherm, dar hinder die fursten ond herren oder die frawen ond junckfr(awen) inner sten, ${ }^{99}$ das man sy mit ainer pirschpuchsen pirschen mag. Von dieser Jagdpraxis vermitteln uns die Jagdbilder Lukas Cranachs d. Ä. von 1544 und 1545 eine so anschauliche Vorstellung, dass ich darauf nicht mehr näher eingehen kann. Wie wir aus dem Reisebericht Herzheimers erfahren, nahm der Kurfürst immer wieder Gäste mit auf die Jagd, nicht nur in Torgau, sondern auch in Lochau, das als Residenzschloss wohl ausschließlich diesem Vergnügen diente.

Fragen wir abschließend nach der Bedeutung der Reiseaufzeichnungen Hans Herzheimers. Selbstzeugnisse, die uns die Perspektive des erlebenden Individuums zeigen, sind um 1500 nicht nur in Mitteldeutschland ausgesprochen selten, wie ich schon einleitend bemerkt habe. Umso dankbarer müssen wir für die Reiseaufzeichnungen Hans Herzheimers sein, auch wenn sie an vielen Stellen knapp sind, manchmal zu knapp. Er selbst bemerkt an verschiedenen Stellen, was er noch alles hätte aufzeichnen können, auch in Torgau: Es wäre gar vil von diser churfurstlichen besytz und ond lustperlichem schonem sloss, so ir churf(urstlich) g(naden) mit frömbden ond newen gepewe erbaben hat, zu schreiben, aber - so fügt er hinzu - So ist es nur zw lang (Editionsanhang Nr. 53).

Doch nicht nur die Kürze mag uns irritieren, sondern auch die spezifische Perspektive dieser Aufzeichnungen, denn Hans Herzheimer hat natürlich eine ganz besondere Brille auf, wenn er durch Kursachsen reitet, und sein Blick dürfte noch durch Reisebegleiter wie Degenhard Pfeffinger in eine ganz bestimmte Richtung gelenkt worden sein. Es war nicht nur zeitgemäßes, sondern auch standesspezifisches Denken, denn die Wahrnehmung adeliger Reisender war selbstverständlich „durch ihre höfische Sozialisation vorgeprägt, auf die eigene Profession hin ausgerichtet“, wie es jüngst Gerhard Fouquet anhand spätmittelalterlicher Berichte von

98 Rudolf Lehmann, Geschichte der Niederlausitz (Veröffentlichungen der Berliner Historischen Kommission 5), Berlin 1963, S. 91.

99 Die Schützen standen hinter einem Schutzschirm. 
adligen Jerusalempilgern herausgearbeitet hat. ${ }^{100}$ Jagd und Krieg, Höfisches jeglicher Art, äußeres Gepränge und Zurschaustellen von Macht, aber auch die Suche nach Abenteuer und Bewährung, das waren wesentliche Aspekte, denen die adligen Reisenden Aufmerksamkeit schenkten. ${ }^{101}$ Die städtisch-bürgerliche Welt interessierte Herzheimer offenkundig ebenso wenig, wie die ländliche Lebenswelt der Bauern. Über die Bürgerstadt Torgau fällt in seinem Reisebericht kein Wort. Das Rathaus mit der dahinter gelegenen Stadtkirche St. Nikolai, das Franziskanerkloster und das städtische Heilig-Geist-Hospital beispielsweise werden mit keinem Wort erwähnt. Sollte er „Torgisch Bier“ getrunken haben (die Altbayern waren um 1500 eher noch Weintrinker), verliert er darüber gleichwohl kein Wort. ${ }^{102}$

Auch die Jerusalempilgerberichte, die bekanntlich die Masse der spätmittelalterlichen Reiseberichte überhaupt ausmachen, sind hilfreich, um die gattungs- und schichtspezifischen Merkmale der Aufzeichnungen Hans Herzheimers, aber auch ihre Besonderheiten zu verstehen. Während die Wahrnehmung der fremden Welt des Heiligen Landes die Verfasser der Pilgerberichte immer wieder anregte, Bezüge zur vertrauten eigenen Lebenswelt herzustellen, ${ }^{103}$ fehlen solche vergleichenden oder kontrastierenden Bemerkungen in Herzheimers Aufzeichnungen gänzlich. Dies dürfte damit zu erklären sein, dass der reisende bayerische Adlige letztlich in Sachsen vieles antraf, was ihm aus seiner Heimat auch vertraut war: eine Welt der Höfe und Residenzen, der Städte und Adelssitze. Man mag die Lücken und die Kürze vieler Schilderungen Hans Herzheimers bedauern, muss vor dem geschilderten Hintergrund zugleich aber auch hervorheben, dass es alles andere als selbstverständlich war, die sächsischen Reiseeindrücke überhaupt aufzuschreiben. Trotz Lücken und einseitiger Perspektiven weiß Hans Herzheimer über die kursächsische Residenz Torgau gleichwohl vieles mitzuteilen, was wir aus anderen Quellen dieser Zeit nicht erfahren. Er war ein einseitiger und doch - wenn er hinschaute - genauer Beobachter. Sein Reisebericht gehört deshalb zu den bedeutendsten Selbstzeugnissen am Übergang vom späten Mittelalter zur Frühen Neuzeit, die über Sachsen berichten.

100 Gerhard Fouquet, Der Reisebericht nach Jerusalem 1494: „Erleben“ - adelige Bewährung und Pilgerschaft, in: Ders. (Hg.), Die Reise eines niederadeligen Anonymus ins Heilige Land im Jahre 1494 (Kieler Werkstücke E/5), Frankfurt a. M. u. a. 2007, S. 24.

$101 \mathrm{Ebd}$.

102 Hans-Joachim Kadatz, Bier und Brauereiwesen in Torgau. Vom Mittelalter bis zum späten 19. Jahrhundert (Schriften des Torgauer Geschichtsvereins 4), Torgau 2001; Jürgen Herzog, Torgauer Bier und der Leipziger Burgkeller (Schriften des Torgauer Geschichtsvereins 9), Beucha 2015.

103 Vgl. hierzu vor allem Esch, Anschauung und Begriff (wie Anm. 92), S. 70-92 und S. 230; Ders., Gemeinsames Erlebnis - individueller Bericht. Vier Parallelberichte aus einer Reisegruppe von Jerusalempilgern 1480, in: Ders., Zeitalter und Menschenalter (wie Anm. 92), S. 189-216 und S. 236 f. Methodisch ähnlich angelegt Ders., Vier Schweizer Parallelberichte von einer Jerusalem-Fahrt im Jahre 1519, in: Ders., Alltag der Entscheidung. Beiträge zur Geschichte der Schweiz an der Wende vom Mittelalter zur Neuzeit, Bern u. a. 1998, S. 355-399. 


\section{Editionsanhang}

Die Beschreibung Torgaus in Hans III. Herzheimers Bericht über seine Reise durch Sachsen 1519.

Überlieferung: Wien, MAK Inv. B.I. 21.517, Standort S20 (Autograf). Die Zäblung der Abschnitte in <> entspricht der von mir herausgegebenen Gesamtedition des Reiseberichts, die voraussichtlich 2017 erscheinen wird.

/fol. 2580:/

$<43>$ Torchaw an der Elbenn, sloss vnd statt a)im landt zw Meissenn, churfurstlich hoffhallta).

Am sonntag nach Erhardi, den 9. tag ianuarii anno 1519 [9. Januar 1519], sein wir von Eylenburg ebens lands durch ainen schonen vorichen vnd aichen walde khomen in ain vesste statt mit schudt vnd polwerck vnd wasser graben pevesstent khomen, genandt Torchaw, ${ }^{104}$ allda ir churf(urstlich) g(naden) auch sein hofflager hellt, vnd in der selben statt hat yr churf(urstlich) g(naden) meinem herrn vnd vettern her(rn) Degnhart Pfeffinger ain schones zirrlichs hawss mit ainem hochen thurn, der vbr all hewß auf geedt, von grundt newes erpawenn. ${ }^{105}$ Es ware lang von dem selben hauss, wie es erpauen ist mit vil schonen stuben, cammern, lussthewseren vnd vil gemachen, hatt mer dann 400 tur, auch ain lustige schone wolgezierte cappellen, padhaws, prunnen, gartten vnd stallen, dar an gar nichts ausgelassen noch von lusstigem haußratt in den chuchellen(?), padgeschirren, silbergeschirren, petgewandt, zyngeschirren, alles aufs raynest aus erfullt, an allen mangl, schon wurtzgertten, nagelstock, so oben auf ettlichen gegigen(?)b) vmbgengen sumerzeit auf mögen gestellt werden. Wer es alles sollte beschreiben, der muest die gemache all von grundt vnden anfachen vnd bis vber das dach auf volenden. $Z_{w}$ negst da selb vber ain gassen ligt vnnser frawen cirichen fast wol erpawen, ${ }^{106}$ dar inn begraben meins $\mathrm{g}$ (nedigen) herrn herzogs Johans von Sachsen etc. elich gemachel fraw Sophia, ain fursstin der gepurdt aus dem fursstenthumb Mecklburg etc. ${ }^{107}$

a) - a) im - hoffhaltt in bellerer Tinte nachgetragen.

b) Über der Zeile, darunter gestrichen zynen.

104 Torgau (Sachsen), an der Elbe.

105 Das Gebäude stand auf dem Grundstück Ritterstraße 10, siehe dazu oben bei Anm. 57.

106 Pfarrkirche St. Marien.

107 Sophia, Tochter Herzog Magnus II. von Mecklenburg, die erste Gemahlin Herzog Johanns des Beständigen, gestorben 1503; siehe Oтто Posse, Die Wettiner. Genealogie des Gesamthauses Wettin Ernestinischer und Albertinischer Linie [...]. Mit Berichtigungen und Ergänzungen der Stammtafeln bis 1993, Leipzig 1994 (erweiterter Nachdruck der Ausgabe Leipzig/Berlin 1897), Tafel 7. Ihre Bronzegrabplatte ist erhalten, siehe oben Anm. 66. 
/fol. 259r:/

$<44>$ Das sloss Torgaw schon erpauen

An der statt eben ligt ain gross sloss, das ist so wunder schon erpawen mit frembden vnd auf ain new artt, der gleichen ich vor nye auf dise artt gesehen hab. Diss sloss gepewe khan ich nit aigentlich beschreiben, wie das mit etlichen hochen knopfen, die vber alle dächer auf sindt gepawen, das man wonnger vnd an alle örtter dar inn vbersehen khan nach allem lusst. Aus dem sloss haben ir chur$\mathrm{f}$ (urstlich) g(naden) ain(en) kunstlichen ganck ${ }^{\mathrm{a}}$ ) bis in die vor ermelt vnnser frawen kirichen gefuerdt, furtter khumbt der selb gangk aus vber die stattmawer vnd statt graben, vber die landstrass, in ain(en) schenen lusstigen pawmgarten. In dem selben gartten haben ir churf(urstlich) g(naden) gepawen zbay schon lussthewß ${ }^{b}$ ) mit raynen lusstigen gemachen vnd aufs kunsstlichest aus malen lassen. Auch dar inne ain lusstiges wildpaldc), die selbig wasserstuben im wildpald ist alle mit zyn aus gemacht, vnd ain lusstige padstuben dabei, vnd was von padgeschier dar ein gehordt, das ist alles von khuppfaren geschieren inwendig rayn ausgezynedt. Da ist kayn geprechen noch mangl, vnd sol man ewig leben. Es ist auch im gartten ain khunstlicher vogel thenne zw dem vinckenfanck gemacht ${ }^{\mathrm{d}}$. Auch dar inne ain lusstige schießhuttene), so man zum zyl schiessen wil, vnd /fol. 259v:/

a) - a) Nota langer ganck am linken Rand nachgetragen. b) Nota lustheuß am linken Rand nachgetragen. c) Nota wildpad am linken Rand nachgetragen. d) Nota vinckenpichl(?) am linken Rand nachgetragen. e) Nota schießhutten am linken Rand nachgetragen.

$<45>$ Trinckstuben im lusthaus

dar vber ain lusstige trinckstubena), vnd aber dar vber noch mer lustige gepew zum aussehen, so man scheusst, oder wan man daselb auch rennedt oder sticht, das alles neben der zylstatt bschehen khan, das mag man alles aus dem selben lussthauss, frawen vnd junckfr(awen), sehen, vnd nichts weniger, khartten, singen, essen oder trincken, oder saytenspill horen, als wär man im paradies. Also ist das selbig lussthaus inwendig mit schoner tappesterei vnd kunstlichen gemall auß beraytt. Vnd neben dem selben gartten fleusst ain gross schiffreiches wasser $\left.{ }^{b}\right)$ fur, genandt die Ellwen, ${ }^{108}$ fleust aus dem land zw Pehaym. Vnd mir ist es vnmöglichn, was irec) churf(urstlich) g(naden) nuch alles hat besehen lassen im sloss vnd in dem gemelten gartten, alles zw beschreiben, yedoch noch ains khan ich nit vnderlassen: In ir churf(urstlich) g(naden) slaff camere zw negst dem grossen schön vnd wolgeziertten furstlichem pedt ist an der ainen seytten gen der wondt, da mein herr vnd lieber vetter ligdt, ain cleynes schones stublend), das ist mit kraußin fladerem holtz alles ausgetafelt, vnd stett dar inn ain schones tischlen. Vnd so obgemelter mein vetter $a b$ dem furstlichem pedt her ab wil, so thutt er an der selben seitten ayn

108 Die Elbe. 
twelen auf vnd khumbt aus dem pedt in dasselb warmes ein gehaytztes schon schtubln vnd thut sich dar inne an. Im sloss sein auch vil grosser hocher /fol. 260r:/

a) Nota trinkstube am linken Rand. b) Nota fliessends wasser am linken Rand. c) i korrigiert. d) Nota stubl pey dem pedt am linken Rand, schon einige Zeilen weiter oben.

$<46>$ Das fursten zymer im sloss

furstlicher zymer, aus den lies ir churf(urstlich) g(naden) ain zymer auf mein gnedigsten herrn, churf(urst) vnd bischowe zw Mayntz etc., ${ }^{109}$ mit costlicher tapossterey auf richten, nemlich vber den furssten tisch 7 gantze stuck von golde nach aller leng oben am poden vnd neben an den wanden costlich niderlendische geworichte seyden tuecher, die zu 600 vnd 800 gulden ${ }^{\text {a) }}$ haben gestanden.

a) Über der Zeile einkorrigiert.

$<47>$ Kirich im sloss

Es haben auch ir churf(urstlich) g(naden) im sloss ain schene kirichena), dar inn vnser frawn tagzeitten taglich gesungen werden. Ir gnad(en) mogen dar ein an zbayen end(en) auf parkirchen khomen. Vnd hat ein schöne orgl.

a) Nota kirich am linken Rand.

$<48>$ Zeughaus im sloss

Es haben ir churf(urstlich) g(naden) auch bey dem sloss ainen grossenn casten mit getraydt oben lygen, vnd vnden mit grossem geschutz, haubttpuchssen, morthen, slänglen vnd menigerlay veldgeschutz ${ }^{a}$.

a) Nota zeughaus am linken Rand.

$<49>$ Visch teicht

Es haben ir churf(urstlich) g(naden) vor dem sloss im velde aynen grossen teicht, den besetzt man mit 3500 schok pruedkarpfen. Vber drey jar vischt man in her aus vnd weegen ye zbennvndreissick 1 center, gibt den center vmb 3 gulden, das lauffet sich auf 3500 gulden, vnd hat dannoch vber hoff ain guet anzal zw speisen.

$<50>$ Kirichlen

Bey bemeltem teicht ligt ain schone klayne wol gezierte kirichen, genandt zum heiligen creutz ${ }^{\mathrm{a}}$, beschehen grosse wunder zaichen, der man vil in der selben

109 Albrecht von Brandenburg, 1513-1545 Erzbischof von Magdeburg und Administrator von Halberstadt, 1514-1545 auch Erzbischof von Mainz, seit 1518 Kardinal; siehe oben Anm. 45. 
kirich siecht. Es ist auch vor der kirichen in aynem grossen pawin ain gross crucifix, das last sich sunsst nindert /fol.260v:/ bringen aus dem pawm, es kumbt als oft man es hat andern enden getragen wider in den selben pawm.

a) Nota heiligencreuz am linken Rand.

$<51>$ Heilig grab

Es hat ir churf(urstlich) g(naden) auf dem selben kirichhoff ain geleichnuss des heiligen grabesa), wie man es zw Jerusalem hatt, auf machen lassen.

a) Nota heilig grab am linken Rand.

$<52>$ Schone pruck

Es haben ir curf(urstlich) g(naden) vber das gross wasser, die Elben, ain kunstliche pruckena) mit gemawerten wasserstuben vnd jochern pawen lassen, als man mich bericht, das die vber 12000 gulden zepawen gestanden hab. Daselbs haben ir churf(urstlich) g(naden) zbay große schyff mit hochen segln auf dem wasser steen vnd sind rayn bedeckt vnd gantz swartz an gestrichen. Daselb sicht man auch große schiff, die von Pehaym her ab komen mit trayd, wein vnd kaufmans guettern.

a) Nota prucken am linken Rand.

$<53>$ Der wald Strudt

Es haben ir churf(urstlich) gnaden jenhalben der selbn prucken zw nagst vil lusstiger jaydea), furnemlichen vnder anderm ist daselb ain schones holtz genant die Strudt, dar auff ist nie anderes wildpratt dann eytl hirschen gefunden. Aber so pald nur vber ain fuerstrass, so hin durch das holtz an das Vngrisch land gen Toberlaw vnd gen Hertzberig gedt, ${ }^{110}$ stendt die hynnd vnd wild vnd hyrsch bey ein ander, b) genandt die khellisch heydt ${ }^{\mathrm{b}}$ ). Ditz jayd behalt ir churf(urstlich) g(naden) auf lieb gesst, dan so man daselb auf der Strud jagt, so lauffn 20 oder mer hirsch auf ain mal herfur, vnd ist so nachent von dem scherm, dar hinder die fursten vnd herren oder die frawen vnd junckfr(awen) inner sten, das man sy mit ainer /fol. 261r:/ pirschpuchsen pirschen mag. Es wäre gar vil von diser churfurstlichen besytz und vnd lustperlichem schonem sloss, so ir churf(urstlich) g(naden) mit frömbden vnd newen gepewe erhaben hat, zu schreiben. So ist es nur zw lang, dan ich pinc) $\mathrm{zw}$ schonen vnd lustigen gepewen furter wordend) gefuert ze sehen, da von ich auch meldung thun wil als her nach voligedt.

a) Nota jayd Strudt am linken Rand. b)-b) Über der Zeile nachgetragen. c) f...g(?) folgt wieder gestrichen. d) $\mathrm{w}$ aus $\mathrm{g}$ korrigiert.

110 Doberlug (Brandenburg) und Herzberg (Brandenburg, an der Elster) in der Niederlausitz, die damals König Ludwig von Böhmen und Ungarn unterstand; siehe oben Anm. 98. 


\section{$[\ldots]$}

$<193>$ Gen Torgaw von Wittenberg komen

An mitichen in osterfeiertagen [27. April 1519] sein magister Ge(org) Spalentinus, meines g(nedig)sten herren caplan, ${ }^{111}$ vnd ich von Wittenberg ausgezogen nach dem frueambt, vnd durcha) ayn kleynes stätlen genandt Thümytz ${ }^{112}$ vnd furter zw vnnser frawen kirichen im Voglgesanck ${ }^{113}$ noch 1 meil bis gen Thorgaw in des her(rn) D(egenhart) Pfeffinger hauss ${ }^{114}$ khomen. Tutt den tag 5 meil.

$\overline{\text { a) d korrigiert. }}$

$<194>$ Am artzet gelegen zu Torgau

Alda zw Torgaw hab ich an meinem pösen payn vor grossem weeclagen nit ferrer faren mögen, vnd byn beij m(eister) Hanns parbier dem aldten ${ }^{115}$, mich wider auf zebringen, in herrn Degnharts hauss beliben vnd maigister(!) Spalentinum zw meinem g(nedi)sten herren gen Grym, ${ }^{116}$ alda ir churf(urstlich) g(naden) die heilig zeit im Augustiner closter sich gehalthen hatt, faren lassen, a) aber ir c. f. g. nitt alda gefunden, wan ir c. f. g. was von Grym widerumb nach Aldenburg khemena).

a) - a) Nachtrag in bellerer Tinte.

$<195>$ Der graff von Nassau

Am pfintztag in osterfeiertagen [28. April 1519] ist mein g(nedig)ster herr gen Altenburg widerumb khemen, alda zw irn c. f. g. der mechtig graffe Henricha) von Nassaw, ${ }^{117}$ mitt yme der margraf Casimirus von Brandenburg, ${ }^{118}$ hertzog Ott der

111 Georg Spalatin; siehe oben Anm. 29.

112 Dommitzsch (Sachsen), an der Elbe.

113 Vogelgesang (Sachsen), Dorf 6,5 km nordwestlich von Torgau; siehe oben Anm. 87.

114 Zum Haus Degenhard Pfeffingers siehe oben bei Anm. 56.

115 Hans d. Ä. Vielleicht ist der bei Ludolphy, Friedrich der Weise (wie Anm. 11), S. 58 erwähnte Torgauer Stadtarzt gemeint?

116 Grimma (Sachsen), an der Mulde.

117 Graf Heinrich III. von Nassau-Breda (1504-1538); KarL E. Demandt, Geschichte des Landes Hessen, revidierter Nachdruck der zweiten, neubearbeiteten und erweiterten Auflage 1972, Kassel 1980, S. 402 f.

118 Markgraf Kasimir von Brandenburg-Ansbach (1481-1527); siehe GÜNTHER ScHuHManN, Kasimir, Markgraf von Brandenburg-Ansbach und -Kulmbach, in: Neue Deutsche Biographie 11 (1977), S. 315 f. 
jung von Lunenburg, ${ }^{119}$ graf Hoyer von Mannsfeld, ${ }^{120}$ her Niclas Ziegler ${ }^{121}$ vnd vil treffenlich ander herren vnd ritter vnd adells, vber 200 personn, khomen sein, alls pottschafftenn kunigs Carls von Hysponia ${ }^{122}$ etc. vnd hertzogenn Ferdinandi aus dem Nidernland Bur(gun)di, ertzherzogen zw Osterreich etc., ${ }^{123}$ vnd haben ir potschafft geworben. /fol. 292r:/ Vnd hatt Herr Niclas Ziegler die werbung mitt schonen zyerlichen wortten vor dem churfurssten gethan, doch ist dise werbung mit versperter thur vnd ir churf(urstlich) g(naden) wenig seiner gehaymbtissten rette da beij gehaltena).

a) In Lücke nachgetragen.

$[\ldots]$

/fol. 292v:/

$[\ldots]$

Am sonntag Misericordias domini den 8. mai [8. Mai 1519] ist mein gnedigster herr zw Eylenburg ${ }^{124}$ auf dem sloss gelegen, vnd am montag darnach [9. Mai 1519] sich gen der Lochaw ${ }^{125}$ erhebt. Alda die potschafft von Wittenberg 5 meil zw iren c. f. g. gen der Lochaw auch khomen. Am erichtag [10. Mai 1519] hat yr c. f. g. die potschafft lassen gen der Sweynitz ${ }^{126}$ an ein schenes hirschgejaid gefuerdt vnd alle khurtzweil vnd ere erpietten lassenn. Am mitwochen [11. Mai 1519] aber lassen ein hirschjaide auf der Lochischen hayd halten, vnd damit gar 3 meil bis gen Torgaw mit ir c. f. g. gefurdt. Am pfintztag [12. Mai 1519] mit der potschafft das frue-

119 Herzog Otto I. von Braunschweig-Lüneburg (1495-1549), der 1520-1527 mit seinen Brüdern das Herzogtum Braunschweig-Lüneburg regierte und dann aufgrund einer morganatischen Ehe mit der Herrschaft Harburg abgefunden wurde; siehe EcKARDT OpITZ, Otto I., in: Hamburgische Biografie, Band 2, Hamburg 2003, S. 311 f. Durch seine Mutter Margarethe von Sachsen war er mit den Wettinern verwandt.

120 Graf Hoyer VI. von Mansfeld-Vorderort (reg. 1484-1540); siehe Jochen Vötsch; Hoyer IV. (VI.), Graf von Mansfeld-Vorderort, in: Sächsische Biografie, hrsg. vom Institut für Sächsische Geschichte und Volkskunde e. V., bearb. von Martina Schattkowsky, Online-Ausgabe: http://www.isgv.de/saebi/ [Zugriff 16. Oktober 2016].

121 Niklas Ziegler (um 1472-um 1527) war seit 1500 oberster Sekretär Kaiser Maximilians I. und wurde auch immer wieder als Gesandter eingesetzt; siehe Hermann WiesFLECKeR, Kaiser Maximilian I. Das Reich, Österreich und Europa an der Wende zur Neuzeit, Band 5: Der Kaiser und seine Umwelt. Hof, Staat, Wirtschaft, Gesellschaft und Kultur, München 1986, S. 254-256.

122 König Karl von Spanien, der künftige Kaiser; siehe Alfred Kohler, Karl V. 15001558. Eine Biographie, München ${ }^{3} 2014$.

123 Ferdinand I. von Habsburg (1503-1564), der Bruder Karls V.; siehe Alfred Kohler, Ferdinand I. 1503-1564. Fürst, König und Kaiser, München 2003.

124 Eilenburg (Sachsen), an der Mulde.

125 Annaburg; siehe oben Anm. 12.

126 Schweinitz (Sachsen-Anhalt). 
mal im pawmgartten gehalten vnd rennen lassen, dar nach a)ist die potschaffta) also von iren c. f. g. von dan abgeschyden, der yrer c. f. g. gaben das gelait auf dem wagen bis zum heiligen creutz, liess sy den tag gen Leibtzigk 6 meyl wider an ir gewär zw iren pferden farenn. /fol. 293r:/

Am freitag [13. Mai 1519] belib ir c. f. g. noch zw Torgaw vnd fertigt her(rn) Niclas Ziegler hin nach, der ritte des tags nach Hall, ${ }^{127}$ von Torgaw 8 meil, aber mein gnedigster herre erholt sich von Torgaw am sambstag [14. Mai 1519], darnach nach Grym, 5 meil, vnd wider en Alldenburg, b) 4 meilb), aber ich Hertzheimer muesste meins bosen payn halben noch zw Torgaw an dem artzedt ligen beleiben. ${ }^{c}$ Aber ir c. f. g. haben treffenlich general getruckt befelh brief lassen ausgeen im landt an all furssten, der ich ainen habc).

Am sonntag Jubilatt, der dritt sonntag nach dem heiligen ostertag [15. Mai 1519], ist zw Leippzig ostermarckt, ${ }^{128}$ bin ich noch zw Torgaw an meinem posen peyn gelegen, wie wol gar vngeren, sonnder vil lieber zw Leibzick auf dem schon marckt geweßen. Am monntag, erichtag, mitichn, pfintztag, freytag vnd samstag [16. bis 21. Mai 1519] noch an dem artzt zw Torchaw gelegen. d)Tutt von Mitichen in osterfeir bis auf montag nach essen 3 wochen 4 tagd).

a)-a) Über der Zeile nachgetragen, darunter gestrichen von den. b)-b) Am linken Rand nachgetragen. c)-c) Nachgetragen. d)-d) Nachgetragen.

$<196>$ Gen Aldenburg widerumb von Torchaw

Am sonntag Cantate, der viert Sonntag [22. Mai 1519], bin ich auf meins g(nedig)sten herren churf(ursten) etc. beuelh gen Aldenburg zw khomen ervodert, den selbn tag meinen sun Johann Jordan von Wittenberg zw mir gen Torgawa) beruefft vnd mit mir gen Aldenburg gefuerdt. Am montag, den 23. tag maij [23. Mai 1519], $\mathrm{zw}$ Torgaw erhoben vnd den tag gen Grym ${ }^{129}$ gefaren, 5 meil, am erichtag [24. Mai 1519] gen Aldenburg, dar nach khomen, 4 meil.

a) T korrigiert.

127 Halle an der Saale (Sachsen-Anhalt).

128 Der Leipziger Ostermarkt, einer der drei jährlichen Markttermine; siehe Markus A. Denzel, Die Leipziger Märkte vom 12. Jahrhundert bis zur Privilegierung von 1497, 1507 und 1514, in: Enno Bünz (Hg.), Geschichte der Stadt Leipzig, Band 1: Von den Anfängen bis zur Reformation, Leipzig 2015, S. 322-340 und S. 855-858, hier S. 333 und S. $337 \mathrm{f}$.

129 Wie Anm. 116. 